\title{
A QUANTIZATION OF BOX-BALL SYSTEMS
}

\author{
R. INOUE, A. KUNIBA, AND M. OKADO
}

\begin{abstract}
An $L$ operator is presented related to an infinite dimensional limit of the fusion $R$ matrices for $U_{q}\left(A_{n-1}^{(1)}\right)$ and $U_{q}\left(D_{n}^{(1)}\right)$. It is factorized into the local propagation operators which quantize the deterministic dynamics of particles and antiparticles in the soliton cellular automata known as the box-ball systems and their generalizations. Some properties of the dynamical amplitudes are also investigated.
\end{abstract}

\section{INTRODUCTION}

The discovery of the box-ball systems [TS, T, TTMS] and their connection to the crystal basis theory [HKT1, HHIKTT, FOY] has led to a new parallelism across the integrable systems of three origins, quantum, ultradiscrete and classical [KOTY2]. They are a class of two dimensional vertex models in statistical mechanics, one dimensional soliton cellular automata and discrete soliton equations. The fundamental objects that govern the local dynamics in these systems are the triad of quantum $R$, combinatorial $R$ and tropical $R$, all satisfying the Yang-Baxter equation. They are a finite dimensional matrix, a bijection among finite sets and a birational map, which are characterized as the intertwiners of $U_{q}$ modules, crystals and geometric crystals, respectively. The box-ball systems $\left(\mathfrak{g}_{n}=A_{n-1}^{(1)}\right)$ and their generalizations to the $\mathfrak{g}_{n}$ automata [HKT1, HKOTY] are associated with the combinatorial $R$, which arises both as the $q \rightarrow 0$ limit of the quantum $R$ and as the ultradiscretization of the tropical $R$ [KOTY1].

An interesting feature in these automata is the factorization of time evolution into a product of propagation operators of particles and antiparticles with fixed color [HKT3, KTT]. This is a consequence of the factorization of the combinatorial $R$ shown in [HKT2]. Our aim in this paper is to elucidate a similar factorization for the relevant quantum $R$, and thereby to launch an integrable quantization of the deterministic dynamics of particles and antiparticles in the generalized box-ball systems.

To illustrate the idea, consider for example the quantum affine algebra $U_{q}\left(A_{n-1}^{(1)}\right)$ and its irreducible finite dimensional representation $V_{m}$ of $m$ fold symmetric tensors. The quantum $R$ matrix for $V_{m} \otimes V_{1}$ (2.3) gives rise to the commuting transfer matrix $T_{m}(z)$ acting on $\cdots \otimes V_{1} \otimes V_{1} \otimes \cdots$, which reduces, at $q=0$, to the time evolution of the box-ball system with capacity $m$ carrier $[\mathrm{TM}]$. One can naturally extract an $L$ operator, a Weyl algebra valued matrix, from the $m \rightarrow \infty$ limit of the $R$ matrix in the vicinity of the lowest weight vector. See (2.13) and (2.14) for example. More general $L$ operators can be constructed similarly corresponding to the $m$ generic situation. The limit considered here is motivated by the box-ball systems and has a special feature in that the resulting $L$ admits the factorization as in Proposition 2.2. Each operator $K_{i}$ appearing there encodes the amplitudes for a local propagation 
of color $i$ particles as depicted in Fig. 2. At $q=0$, it reduces to the deterministic dynamics in the box-ball system $[\mathrm{T}]$.

Sections 2.1-2.6 are devoted to an exposition of these observations. Sections 2.7 and 2.8 are concerned with some properties of the dynamical amplitudes and the implication of the Bethe ansatz, respectively. In section 3 we establish parallel results on $D_{n}^{(1)}$ case. The calculation of the fusion $R \in \operatorname{End}\left(V_{m} \otimes V_{1}\right)$ is more involved than $A_{n-1}^{(1)}$. It is done in the limit $m \rightarrow \infty$ in appendix A. The $L$ operator is given in section 3.3 and factorized in section 3.4. The propagation operators describe the amplitudes of pair creation and annihilation of particles and antiparticles as depicted in Fig. 8. A quantized $D_{n}^{(1)}$ automaton is presented in section 3.5 with a few basic properties.

The fusion construction of the $R$ matrices and their matrix elements for $A_{n-1}^{(1)}$ given in section 2 are not new. They have been included for the sake of selfcontainment. The content of this paper may be regarded as a generalization of the one in [HKT2] for $q=0$. It will be interesting to investigate the present results in the light of the works $[\mathrm{KT}, \mathrm{KR}, \mathrm{S}]$.

\section{2. $A_{n-1}^{(1)} \mathrm{CASE}$}

2.1. $\quad \boldsymbol{R}$ matrix $\boldsymbol{R}(\boldsymbol{z})$ and its fusion $\boldsymbol{R}^{(\boldsymbol{m}, \mathbf{1})}(\boldsymbol{z})$. We recall the standard fusion construction [KRS]. Let $V=\mathbb{C} v_{1} \oplus \cdots \oplus \mathbb{C} v_{n}$ be the vector representation of the quantum affine algebra $U_{q}=U_{q}\left(A_{n-1}^{(1)}\right)$ without the derivation operator. Here $v_{1}$ is the highest weight vector and our convention of the coproduct is $\Delta\left(e_{i}\right)=$ $e_{i} \otimes 1+t_{i} \otimes e_{i}, \Delta\left(f_{i}\right)=f_{i} \otimes t_{i}^{-1}+1 \otimes f_{i}$ for the Chevalley generators. The $R$ matrix $R(z) \in \operatorname{End}(V \otimes V)$ reads

$$
\begin{aligned}
& R(z)=a(z) \sum_{i} E_{i i} \otimes E_{i i}+b(z) \sum_{i \neq j} E_{i i} \otimes E_{j j}+c(z)\left(z \sum_{i<j}+\sum_{i>j}\right) E_{j i} \otimes E_{i j}, \\
& a(z)=1-q^{2} z, \quad b(z)=q(1-z), \quad c(z)=1-q^{2},
\end{aligned}
$$

where $E_{i j}$ is the matrix unit acting as $E_{i j} v_{k}=\delta_{j k} v_{i}$. It satisfies the Yang-Baxter equation $R_{23}\left(z^{\prime} / z\right) R_{13}\left(z^{\prime}\right) R_{12}(z)=R_{12}(z) R_{13}\left(z^{\prime}\right) R_{23}\left(z^{\prime} / z\right)$. The matrix $\check{R}(z)=$ $P R(z)$ commutes with $\Delta\left(U_{q}\right)$, where $P$ denotes the transposition of the components.

Let $V_{m}$ be the irreducible $U_{q}$ module spanned by the $m$ fold $q$-symmetric tensors. We take $V_{1}=V$ and realize the space $V_{m}$ as the quotient $V^{\otimes m} / A$, where $A=$ $\sum_{j} V^{\otimes j} \otimes \operatorname{Im} P R\left(q^{-2}\right) \otimes V^{\otimes m-2-j}$. It is easy to see $\operatorname{Im} P R\left(q^{-2}\right)=\operatorname{Ker} P R\left(q^{2}\right)=$ $\bigoplus_{i<j} \mathbb{C}\left(v_{i} \otimes v_{j}-q v_{j} \otimes v_{i}\right)$. For $n \geq i_{1} \geq \cdots \geq i_{m} \geq 1$, we write the vector $\left(v_{i_{1}} \otimes \cdots \otimes v_{i_{m}} \bmod A\right) \in V_{m}$ as $x=\left[x_{1}, \ldots, x_{n}\right]$, where $x_{i}$ is the number of the letter $i$ in the sequence $i_{1}, \ldots, i_{m}$. Thus, $x_{i} \in \mathbb{Z}_{\geq 0}$ and $x_{1}+\cdots+x_{n}=m$ holds.

Due to the Yang-Baxter equation, the operator

$$
\frac{R_{1, m+1}\left(z q^{m-1}\right) R_{2, m+1}\left(z q^{m-3}\right) \cdots R_{m, m+1}\left(z q^{-m+1}\right)}{a\left(z q^{m-3}\right) a\left(z q^{m-5}\right) \cdots a\left(z q^{-m+1}\right)}
$$


FIGURE 1. Diagram for $w_{j k}[x \mid y]$

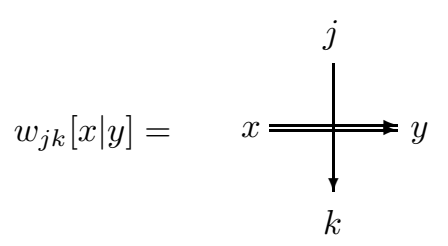

can be restricted to $\operatorname{End}\left(V_{m} \otimes V\right)$. As a result we get an $m$ by 1 fusion $R$ matrix $R^{(m, 1)}(z) \in \operatorname{End}\left(V_{m} \otimes V\right)$, which reads explicitly as

$$
\begin{aligned}
& R^{(m, 1)}(z)\left(x \otimes v_{j}\right)=\sum_{k} w_{j k}[x \mid y]\left(y \otimes v_{k}\right), \\
& w_{j k}[x \mid y]= \begin{cases}q^{m-x_{k}}-q^{x_{k}+1} z & j=k \\
\left(1-q^{2 x_{k}}\right) q^{x_{k+1}+x_{k+2}+\cdots+x_{j-1}} z & j>k \\
\left(1-q^{2 x_{k}}\right) q^{m-\left(x_{j}+x_{j+1}+\cdots+x_{k}\right)} & j<k .\end{cases}
\end{aligned}
$$

It is customary to attach the matrix element $w_{j k}[x \mid y]$ with a diagram like Fig. 1. Here $y=\left[y_{i}\right]$ is specified by the weight conservation as

$$
y_{i}=x_{i}+\delta_{i j}-\delta_{i k}
$$

in terms of $x, j$ and $k$. At $q=0$, the matrix element $w_{j k}[x \mid y]$ is nonzero if and only if $x \otimes v_{j} \simeq v_{k} \otimes y$ in the combinatorial $R: B_{m} \otimes B_{1} \simeq B_{1} \otimes B_{m}$, where it takes the value $z^{H}$, with $1-H=$ winding number [NY]. The fusion $R$ matrix $R^{(m, 1)}(z)$ reduces to $R(z)$ in (2.1) for $m=1$, and it satisfies the Yang-Baxter equation in $\operatorname{End}\left(V_{m} \otimes V \otimes V\right):$

$$
R_{23}\left(z^{\prime} / z\right) R_{13}^{(m, 1)}\left(z^{\prime}\right) R_{12}^{(m, 1)}(z)=R_{12}^{(m, 1)}(z) R_{13}^{(m, 1)}\left(z^{\prime}\right) R_{23}\left(z^{\prime} / z\right)
$$

The $R$ matrix $R^{(1, m)}(z) \in \operatorname{End}\left(V \otimes V_{m}\right)$ is similarly obtained as $R^{(1, m)}(z)\left(v_{j} \otimes\right.$ $x)=\sum_{k} \bar{w}_{j k}[x \mid y]\left(v_{k} \otimes y\right)$, where

$$
\bar{w}_{j k}[x \mid y]= \begin{cases}q^{m-x_{k}}-q^{x_{k}+1} z & j=k \\ \left(1-q^{2 x_{k}}\right) q^{m-\left(x_{k}+x_{k+1}+\cdots+x_{j}\right)} & j>k \\ \left(1-q^{2 x_{k}}\right) q^{x_{j+1}+x_{j+2}+\cdots+x_{k-1}} z & j<k .\end{cases}
$$

The inversion relation

$$
P R^{(1, m)}\left(z^{-1}\right) P R^{(m, 1)}(z)=\left(1-q^{m+1} z\right)\left(1-q^{m+1} z^{-1}\right) \mathrm{Id}
$$

is valid.

2.2. $\boldsymbol{L}$ operator $\boldsymbol{L}(\boldsymbol{z})$. Now we extract an $L$ operator $L(z)$ from a certain limit of $R^{(m, 1)}(z)$. We illustrate the idea along the $n=3$ case. The 3 by 3 matrix $\left(w_{j i}[x \mid y]\right)_{1 \leq i, j \leq 3}$ with $y$ chosen as (2.5) looks as

$$
\left(\begin{array}{ccc}
q^{x_{2}+x_{3}}-q^{x_{1}+1} z & \left(1-q^{2 x_{1}}\right) z & \left(1-q^{2 x_{1}}\right) q^{x_{2}} z \\
\left(1-q^{2 x_{2}}\right) q^{x_{3}} & q^{x_{1}+x_{3}}-q^{x_{2}+1} z & \left(1-q^{2 x_{2}}\right) z \\
1-q^{2 x_{3}} & \left(1-q^{2 x_{3}}\right) q^{x_{1}} & q^{x_{1}+x_{2}}-q^{x_{3}+1} z
\end{array}\right) .
$$

Throughout the paper we assume that $|q|<1$. Consider the limit $m \rightarrow \infty$ with $x_{1}$ and $x_{2}$ kept fixed. Namely we take $x_{3} \rightarrow \infty$ and stay in the vicinity of the lowest 
weight vector of $V_{m}$ as $m$ goes to infinity. The above matrix simplifies to

$$
\left(\begin{array}{ccc}
-q^{x_{1}+1} z & \left(1-q^{2 x_{1}}\right) z & \left(1-q^{2 x_{1}}\right) q^{x_{2}} z \\
0 & -q^{x_{2}+1} z & \left(1-q^{2 x_{2}}\right) z \\
1 & q^{x_{1}} & q^{x_{1}+x_{2}}
\end{array}\right)
$$

In the limit, the constraint $x_{1}+x_{2} \leq m$ becomes void and the vector $x=$ $\left[x_{1}, x_{2}, x_{3}\right] \in V_{m}$ gets effectively labeled as $\left[x_{1}, x_{2}\right]$ with arbitrary $x_{1}, x_{2} \in \mathbb{Z}_{\geq 0}$. For generic (nonzero) $x_{1}$ and $x_{2}$, the $(1,2)$ element $\left(1-q^{2 x_{1}}\right) z$ in $(2.8)$, for example, is the matrix element of the transition $\left[x_{1}, x_{2}\right] \rightarrow\left[x_{1}-1, x_{2}+1\right]$ in view of (2.5). Similarly the $(2,3)$ element $\left(1-q^{2 x_{2}}\right) z$ is the one for $\left[x_{1}, x_{2}\right] \rightarrow\left[x_{1}, x_{2}-1\right]$. Introducing the operator $P_{2}$ and $Q_{2}$ that act on $\left[x_{1}, x_{2}\right]$ as $P_{2}\left[x_{1}, x_{2}\right]=q^{x_{2}}\left[x_{1}, x_{2}\right]$ and $Q_{2}\left[x_{1}, x_{2}\right]=\left[x_{1}, x_{2}+1\right]$, the $(2,3)$ element of $(2.8)$ is represented as $z Q_{2}^{-1}\left(1-P_{2}^{2}\right)$. With the similar operators $P_{1}$ and $Q_{1}$ concerning the coordinate $x_{1}$, the matrix (2.8) is presented as

$$
\left(\begin{array}{ccc}
-z q P_{1} & z Q_{1}^{-1}\left(1-P_{1}^{2}\right) Q_{2} & z Q_{1}^{-1}\left(1-P_{1}^{2}\right) P_{2} \\
0 & -z q P_{2} & z Q_{2}^{-1}\left(1-P_{2}^{2}\right) \\
Q_{1} & P_{1} Q_{2} & P_{1} P_{2}
\end{array}\right) .
$$

where operators are all commutative except $P_{i} Q_{i}=q Q_{i} P_{i}$.

Motivated by these observations, we prepare for general $n$ the Weyl algebra generated by the pairs $P_{i}^{ \pm 1}, Q_{i}^{ \pm 1}(1 \leq i \leq n-1)$ under the relations

$$
\begin{aligned}
& Q_{i} Q_{j}=Q_{j} Q_{i}, \quad P_{i} P_{j}=P_{j} P_{i}, \quad P_{i} Q_{j}=q^{\delta_{i j}} Q_{j} P_{i}, \\
& Q_{i} Q_{i}^{-1}=Q_{i}^{-1} Q_{i}=1, \quad P_{i} P_{i}^{-1}=P_{i}^{-1} P_{i}=1 .
\end{aligned}
$$

We actually consider a slight generalization of (2.9) containing parameters $a_{1}, \ldots, a_{n-1}$. Let $\mathcal{A}$ be the subalgebra of the Weyl algebra generated by

$$
P_{i}, \quad Q_{i}, \quad R_{i}=Q_{i}^{-1}\left(1-a_{i} P_{i}^{2}\right) \quad 1 \leq i \leq n-1 .
$$

We also use the subsidiary symbol $P_{i}^{\prime}=-a_{i} q P_{i}$. The previous discussion corresponds to $\forall a_{i}=1$ case. The combination $R_{i} \in \mathcal{A}$ introduced here should not be confused with the $R$ matrix. Then we define the operator $L(z) \in \mathcal{A} \otimes \operatorname{End}(V)$ by

$$
L(z)=\left(\begin{array}{ccc}
L_{11}(z) & \cdots & L_{1 n}(z) \\
\vdots & \ddots & \vdots \\
L_{n 1}(z) & \cdots & L_{n n}(z)
\end{array}\right)
$$

where $L_{i j}(z) \in \mathcal{A}$ is given by $\left(P_{i, j}=P_{i} P_{i+1} \cdots P_{j}\right.$ for $\left.i \leq j\right)$

$$
L(z)_{i i}=\left\{\begin{array}{ll}
z P_{i}^{\prime} & i<n, \\
P_{1, n-1} & i=n,
\end{array} \quad L(z)_{i j}= \begin{cases}z R_{i} P_{i+1, j-1} Q_{j} & i<j<n, \\
z R_{i} P_{i+1, n-1} & i<j=n, \\
P_{1, j-1} Q_{j} & j<i=n, \\
0 & j<i<n .\end{cases}\right.
$$

This is an operator interpretation of $w_{j i}[x \mid y](2.4)$ in the limit $x_{n} \rightarrow \infty$ deformed with $a_{1}, \ldots, a_{n-1}$. See (2.24). For example for $A_{1}^{(1)}$ and $A_{2}^{(1)}$, they read

$$
L(z)=\left(\begin{array}{cc}
z P_{1}^{\prime} & z R_{1} \\
Q_{1} & P_{1}
\end{array}\right), \quad L(z)=\left(\begin{array}{ccc}
z P_{1}^{\prime} & z R_{1} Q_{2} & z R_{1} P_{2} \\
0 & z P_{2}^{\prime} & z R_{2} \\
Q_{1} & P_{1} Q_{2} & P_{1,2}
\end{array}\right) .
$$


The latter agrees with $(2.9)$ when $\forall a_{i}=1$. For $A_{3}^{(1)}$ one has

$$
L(z)=\left(\begin{array}{cccc}
z P_{1}^{\prime} & z R_{1} Q_{2} & z R_{1} P_{2} Q_{3} & z R_{1} P_{2,3} \\
0 & z P_{2}^{\prime} & z R_{2} Q_{3} & z R_{2} P_{3} \\
0 & 0 & z P_{3}^{\prime} & z R_{3} \\
Q_{1} & P_{1} Q_{2} & P_{1,2} Q_{3} & P_{1,3}
\end{array}\right)
$$

Our convention is $L(z)\left(\alpha \otimes v_{j}\right)=\sum_{i}\left(L_{i j}(z) \alpha\right) \otimes v_{i}$ for $\alpha \in \mathcal{A}$. Similarly we let $\stackrel{1}{L}(z), \stackrel{2}{L}(z) \in \mathcal{A} \otimes \operatorname{End}(V \otimes V)$ denote the operators acting as $\stackrel{1}{L}(z)\left(\alpha \otimes v_{i} \otimes v_{j}\right)=$ $\sum_{k}\left(L_{k i}(z) \alpha\right) \otimes v_{k} \otimes v_{j}$ and $\stackrel{2}{L}(z)\left(\alpha \otimes v_{i} \otimes v_{j}\right)=\sum_{k}\left(L_{k j}(z) \alpha\right) \otimes v_{i} \otimes v_{k}$. As an analogue of the Yang-Baxter equation (2.6), we have

\section{Proposition 2.1.}

$$
R\left(z_{2} / z_{1}\right) \stackrel{2}{L}\left(z_{2}\right) \stackrel{1}{L}\left(z_{1}\right)=\stackrel{1}{L}\left(z_{1}\right) \stackrel{2}{L}\left(z_{2}\right) R\left(z_{2} / z_{1}\right) \in \mathcal{A} \otimes \operatorname{End}(V \otimes V) .
$$

In section 2.3 , this will be proved based on the factorization of $L(z)$.

2.3. Factorization of $\boldsymbol{L}(\boldsymbol{z})$. Let us introduce the operators $K_{i} \in \mathcal{A} \otimes \operatorname{End}(V)$ for $1 \leq i \leq n-1$ by

$$
\begin{aligned}
& K_{i}=\left(\left(K_{i}\right)_{j, k}\right)_{1 \leq j, k \leq n}, \\
& \left(K_{i}\right)_{i, i}=P_{i}^{\prime},\left(K_{i}\right)_{i, n}=R_{i},\left(K_{i}\right)_{n, i}=Q_{i},\left(K_{i}\right)_{n, n}=P_{i}, \\
& \left(K_{i}\right)_{j, j}=1(j \neq i, n) .
\end{aligned}
$$

The other elements are zero. The $K_{i}$ with $\forall a_{i}=1$ will be interpreted as the local propagation operator in quantized box-ball system in section 2.6. We also introduce an $n$ by $n$ matrix

which acts on $V$ only.

$$
D(z)=z \operatorname{diag}\left(1, \ldots, 1, z^{-1}\right),
$$

\section{Proposition 2.2.}

$$
L(z)=D(z) K_{1} K_{2} \cdots K_{n-1}
$$

For example the latter in (2.13) is expressed as

$$
\left(\begin{array}{ccc}
z P_{1}^{\prime} & z R_{1} Q_{2} & z R_{1} P_{2} \\
0 & z P_{2}^{\prime} & z R_{2} \\
Q_{1} & P_{1} Q_{2} & P_{1} P_{2}
\end{array}\right)=\operatorname{diag}(z, z, 1)\left(\begin{array}{ccc}
P_{1}^{\prime} & 0 & R_{1} \\
0 & 1 & 0 \\
Q_{1} & 0 & P_{1}
\end{array}\right)\left(\begin{array}{ccc}
1 & 0 & 0 \\
0 & P_{2}^{\prime} & R_{2} \\
0 & Q_{2} & P_{2}
\end{array}\right) .
$$

Proof. Denote the $n$ by $n$ matrix $L(z=1)$ defined by (2.12) by $L_{n}$. We are to show $K_{1} K_{2} \ldots K_{n-1}=L_{n}$ for $A_{n-1}^{(1)}$. This is done by induction on $n$. The case $n=3$ is checked in the above. Suppose the equality is valid for $n$. Then from the structure of the matrices $K_{i}$, one can evaluate $K_{1} K_{2} \cdots K_{n}$ for $A_{n}^{(1)}$ as the product of $K_{1}$ and the rest as

$$
\left(\begin{array}{ccc}
P_{1}^{\prime} & & R_{1} \\
& & \\
& \mathbb{1}_{n-1} & \\
Q_{1} & & P_{1}
\end{array}\right)\left(\begin{array}{cccc}
1 & 0 & \cdots & 0 \\
0 & & & \\
\vdots & & L_{n}^{+} & \\
0 & & &
\end{array}\right)=L_{n+1} .
$$

Here $L_{n}^{+}$is $L_{n}$ with all the constituent operators $X_{i}\left(X=P, P^{\prime}, Q, R\right)$ replaced by $X_{i+1}$, and $\mathbb{1}_{n-1}$ is the identity matrix of size $n-1$. It is straightforward to verify this identity. 
Remark 2.3. Elements of $\mathcal{A}$ contained in any single $L_{i j}(z)(2.12)$ are all commutative. As a result, the identity (2.16) holds under any interchange of $P_{i}, P_{i}^{\prime}, Q_{i}$ and $R_{i}$ on the both sides.

Let us make use of the factorization to prove Proposition 2.1. We first define $\sigma_{1}, \ldots, \sigma_{n-1}, \sigma \in \operatorname{End}(V)$ by

$$
\begin{aligned}
& \sigma_{i} v_{j}= \begin{cases}v_{i+1} & \text { if } j=i \\
v_{i} & \text { if } j=i+1 \\
v_{j} & \text { otherwise }\end{cases} \\
& \sigma=\sigma_{n-1} \sigma_{n-2} \cdots \sigma_{1} .
\end{aligned}
$$

Thus $\sigma v_{j}=v_{j-1}$ is valid for indices in $\mathbb{Z} / n \mathbb{Z}$. Consider the following gauge transformation of $K_{i}$ :

$$
S_{i}=\sigma_{i} \sigma_{i+1} \cdots \sigma_{n-1} K_{i} \sigma_{n-1} \sigma_{n-2} \cdots \sigma_{i+1} \quad 1 \leq i \leq n-1 .
$$

The components of $S_{i} \in \mathcal{A} \otimes \operatorname{End}(V)$ are given by

$$
\begin{aligned}
& S_{i}=\left(\left(S_{i}\right)_{j, k}\right)_{1 \leq j, k \leq n}, \\
& \left(S_{i}\right)_{i, i+1}=P_{i},\left(S_{i}\right)_{i+1, i+1}=R_{i},\left(S_{i}\right)_{i, i}=Q_{i},\left(S_{i}\right)_{i+1, i}=P_{i}^{\prime}, \\
& \left(S_{i}\right)_{j, j}=1(j \neq i, i+1) .
\end{aligned}
$$

The other components are zero. Note that Proposition 2.2 is rewritten as

$$
L(z)=D(z) \sigma S_{1} S_{2} \cdots S_{n-1} .
$$

Now Proposition 2.1 is a corollary of the formula (2.19) and

\section{Lemma 2.4 .}

$$
\begin{aligned}
& R\left(z_{2} / z_{1}\right)\left(D\left(z_{1}\right) \sigma \otimes D\left(z_{2}\right) \sigma\right)=\left(D\left(z_{1}\right) \sigma \otimes D\left(z_{2}\right) \sigma\right) R\left(z_{2} / z_{1}\right) \\
& 2 \stackrel{1}{2} \stackrel{1}{2}_{S} S_{i}=S_{i} R(z) \quad 1 \leq i \leq n-1 .
\end{aligned}
$$

Proof. The first relation is directly confirmed. It is enough to check the latter at two distinct values of $z$. It is trivially valid at $z=1$ and easily checked at $z=q^{-2}$.

Remark 2.5. If $a_{i}=1$, the property

$$
K_{i}^{2}=\mathbb{1}_{n}
$$

is valid for $1 \leq i \leq n-1$. This is a remnant of the inversion relation (2.7). It implies $L(z)^{-1}=K_{n-1} \cdots K_{1} D\left(z^{-1}\right)$. The formula (2.19) was known at $q=0$ as a factorization of combinatorial $R$ [HKT2], where $S_{i}$ appeared as the Weyl group operator on crystal basis.

For $A_{1}^{(1)}$, the $L$ operator here can also be obtained by specializing the $q$ generic case of the one in [BS]. The case $\forall a_{i}=0$ has appeared in the quantized Volterra model for $A_{n-1}^{(1)}[\mathrm{HIK}]$. 
2.4. Quantized box-ball system: Space of states. Consider the formal infinite tensor product of $V=\mathbb{C} v_{1} \oplus \cdots \oplus \mathbb{C} v_{n}$ :

$$
\cdots \otimes V \otimes V \otimes V \otimes \cdots=\oplus \mathbb{C}\left(\cdots \otimes v_{j_{-1}} \otimes v_{j_{0}} \otimes v_{j_{1}} \otimes \cdots\right)
$$

An element of the form $c\left(\cdots \otimes v_{j_{-1}} \otimes v_{j_{0}} \otimes v_{j_{1}} \otimes \cdots\right)$ will be called a monomial (a monic monomial if with $c=1$ ). The space of states of our quantized box-ball system is the subspace of (2.20) given by

$$
\mathcal{P}=\left\{\sum_{p: \text { monic monomial }} c_{p} p \mid \text { conditions (i) and (ii) }\right\},
$$

where (i) $\sum_{k \in \mathbb{Z}}\left|j_{k}-n\right|<\infty$ for any $p=\cdots \otimes v_{j_{-1}} \otimes v_{j_{0}} \otimes v_{j_{1}} \otimes \cdots$ appearing in the sum, (ii) there exists $N \in \mathbb{Z}$ such that $\lim _{q \rightarrow 0} q^{N} \sum_{p} c_{p} p=0$. Monomials can be classified according to the numbers $w_{1}, \ldots, w_{n-1}$ of occurrence of the letters $1, \ldots, n-1$ in the set $\left\{j_{k}\right\}$. Consequently one has the direct sum decomposition:

$$
\mathcal{P}=\oplus \mathcal{P}_{w_{1}, w_{2}, \ldots, w_{n-1}},
$$

where the sum runs over $\left(w_{1}, \ldots, w_{n-1}\right) \in \mathbb{Z}_{\geq 0}^{n-1}$. We have $\mathcal{P}_{0, \ldots, 0}=\mathbb{C} p_{\text {vac }}$, where $p_{\text {vac }}=\cdots \otimes v_{n} \otimes v_{n} \otimes \cdots$.

The local states $v_{j_{k}} \in V$ is regarded as the $k$ th box containing a ball with color $j_{k}$ if $j_{k} \neq n$, and the empty box if $j_{k}=n$. The space of states of the box-ball system is the totality of the monomials in the above sense. The space of states $\mathcal{P}$ of our quantized box-ball system consists of linear superpositions thereof.

2.5. Time evolution. We set $\forall a_{i}=1$ in the remainder of section 2. Then the following provides an $\mathcal{A}$ module $\mathcal{M}$ :

$$
\begin{aligned}
& \mathcal{M}=\oplus_{m_{1}, \ldots, m_{n-1} \in \mathbb{Z}_{\geq 0}} \mathbb{C}\left[m_{1}, \ldots, m_{n-1}\right], \\
& P_{i}\left[\ldots, m_{i}, \ldots\right]=q^{m_{i}}\left[\ldots, m_{i}, \ldots\right], \\
& Q_{i}\left[\ldots, m_{i}, \ldots\right]=\left[\ldots, m_{i}+1, \ldots\right], \\
& R_{i}\left[\ldots, m_{i}, \ldots\right]=\left(1-q^{2 m_{i}}\right)\left[\ldots, m_{i}-1, \ldots\right],
\end{aligned}
$$

where the right hand side of the last formula is to be understood as 0 at $m_{i}=0$. The space $\mathcal{M}$ will be regarded as the space of the quantum carrier. By construction, for $x=\left[x_{1}, \ldots, x_{n-1}\right] \in \mathcal{M}$ one has

$$
\begin{aligned}
& L(z)\left(x \otimes v_{j}\right)=\sum_{k} W_{j k}[x \mid y]\left(y \otimes v_{k}\right), \\
& W_{j k}[x \mid y]=\lim _{x_{n} \rightarrow \infty} w_{j k}\left[x_{1}, \ldots, x_{n-1}, x_{n} \mid y_{1}, \ldots, y_{n-1}, y_{n}\right],
\end{aligned}
$$

where $y$ is determined from (2.5) in terms of $j, k$ and $x$.

According to the standard construction of transfer matrices in two dimensional solvable vertex models [Bax], the time evolution $T(z): \mathcal{P} \rightarrow \mathcal{P}$ is constructed as a composition of local $L$ operators as

$$
T(z)=(\cdots \stackrel{1}{L}(z) \stackrel{0}{L}(z) \stackrel{-1}{L}(z) \cdots)_{0,0} .
$$

Here $\stackrel{k}{L}(z) \in \operatorname{End}(\mathcal{M} \otimes \mathcal{P})$ signifies the representation of the $L$ operator:

$$
\begin{aligned}
& \stackrel{k}{L}(z)\left(m \otimes\left(\cdots \otimes v_{j_{k-1}} \otimes v_{j_{k}} \otimes v_{j_{k+1}} \otimes \cdots\right)\right) \\
& =\sum_{i}\left(L_{i j_{k}}(z) m\right) \otimes\left(\cdots \otimes v_{j_{k-1}} \otimes v_{i} \otimes v_{j_{k+1}} \otimes \cdots\right),
\end{aligned}
$$


where $L_{i j_{k}}(z) m$ for $m \in \mathcal{M}$ is specified by (2.23). The symbol $(\cdots)_{0,0}$ in $(2.25)$ stands for the element in $\operatorname{End}(\mathcal{P})$ that is attached to the transition $[0, \ldots, 0] \mapsto$ $[0, \ldots, 0]$ in the $\mathcal{M}$ part. By the definition $T(z)$ preserves the weight subspace $\mathcal{P}_{w_{1}, w_{2}, \ldots, w_{n-1}}$ and acts homogeneously on it as

$$
T(z) p=z^{w_{1}+\cdots+w_{n-1}} T(1) p \quad \text { for } p \in \mathcal{P}_{w_{1}, w_{2}, \ldots, w_{n-1}} .
$$

Therefore the commutativity $T(z) T\left(z^{\prime}\right)=T\left(z^{\prime}\right) T(z)$ is trivially valid. Henceforth we concentrate on $T=T(z=1)$, and $T(p)$ for $p \in \mathcal{P}$ is to be understood as $T(1) p$.

2.6. Factorized dynamics. The time evolution $T$ admits a simple description as the product of propagation operators. Set

$$
\mathcal{K}_{i}=\left(\cdots \stackrel{1}{K_{i}} \stackrel{0}{K}_{i} \stackrel{-1}{K}_{i} \cdots\right)_{0,0} \in \operatorname{End}(\mathcal{P}) \quad 1 \leq i \leq n-1,
$$

where the representation $\stackrel{k}{K}{ }_{i} \in \operatorname{End}(\mathcal{M} \otimes \mathcal{P})$ is specified from $K_{i}(2.15)$ in the same way as $\stackrel{k}{L}(z)$ was done via $L(z)$. To interpret $\mathcal{K}_{i}$ pictorially, we attach the following diagrams to the local operator $K_{i}$.

FiguRE 2. Diagram for $K_{i}(j \neq i, n)$

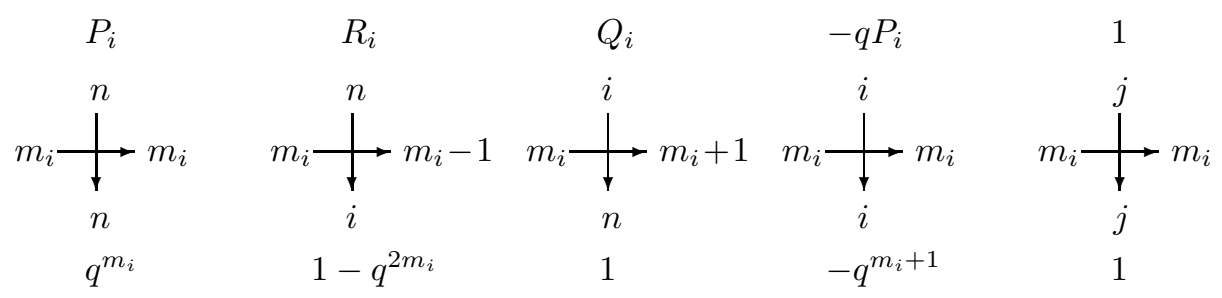

Here $m_{i} \in \mathbb{Z}_{\geq 0}$ is a coordinate in $\left[m_{1}, \ldots, m_{n-1}\right] \in \mathcal{M}$. The horizontal and vertical arrows correspond to $\mathcal{M}$ and $V$, respectively. The diagrams depict the interaction between the local box and the quantum carrier containing $m_{i}$ balls of color $i$. The carrier coming from the left encounters the local box whose state are specified on the top. It picks up/down a color $i$ ball or does nothing and proceeds to the right leaving the box in the state given in the bottom with the listed amplitudes. The first line in the figure gives the operators acting on $\mathcal{M}$ that yield the amplitudes on the last line. For example one has

$$
\begin{aligned}
K_{i}\left(\left[\ldots, m_{i}, \ldots\right] \otimes v_{n}\right) & =\left(P_{i}\left[\ldots, m_{i}, \ldots\right]\right) \otimes v_{n}+\left(R_{i}\left[\ldots, m_{i}, \ldots\right]\right) \otimes v_{i} \\
& =q^{m_{i}}\left[\ldots, m_{i}, \ldots\right] \otimes v_{n}+\left(1-q^{2 m_{i}}\right)\left[\ldots, m_{i}-1, \ldots\right] \otimes v_{i} .
\end{aligned}
$$

The second term describes unloading whereas the first term is just a passage. It is easy to see that at $q=0, K_{i}$ reduces to the deterministic operator which coincides with the local interaction between a carrier and a box $[\mathrm{TM}]$ in the conventional box-ball system [T, TS]. Now the composition (2.28) is expressed as Fig. 3.

The amplitude of $\mathcal{K}_{i}$ assigned with the transition from $\left(\cdots \otimes v_{j_{0}} \otimes v_{j_{1}} \otimes \cdots\right)$ to $\left(\cdots \otimes v_{i_{0}} \otimes v_{i_{1}} \otimes \cdots\right)$ is obtained as the product of all the amplitudes attached to the local vertices in Fig. 3 according to the rule specified in Fig. 2. The calculation involves an infinite product, which is well defined for elements in $\mathcal{P}$. See section 2.7 for examples of computations of the amplitudes. 
FiguRE 3. Diagram for $\mathcal{K}_{i}(j \neq i, n)$

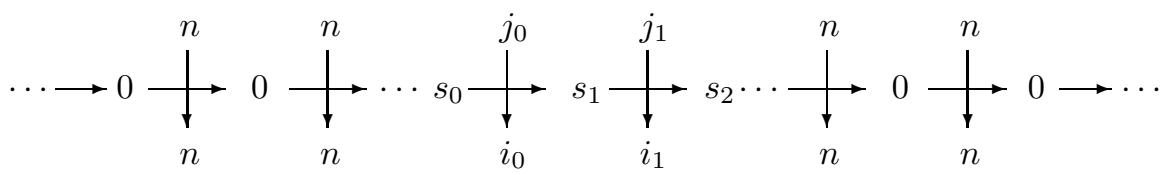

Theorem 2.6. The time evolution of the quantized box-ball system admits a factorization into propagation operators as

$$
T=\mathcal{K}_{1} \cdots \mathcal{K}_{n-1} .
$$

Proof. This is a consequence of the definitions (2.25), (2.28) and the factorization of the $L$ operator established in Proposition 2.2.

At $q=0$, Theorem 2.6 reduces to the original description of the time evolution in the box-ball system $[\mathrm{T}]$ as the composition of finer process to move balls with a fixed color.

2.7. Some properties of amplitudes. For simplicity we concentrate on $A_{1}^{(1)}$ case in the remainder of section 2 , where one only has one kind of ball and $T=\mathcal{K}_{1}$. However, by virtue of Theorem 2.6, all the essential statements are equally valid for general $A_{n-1}^{(1)}$ under an appropriate resetting. In particular, Proposition 2.7 and Proposition 2.9 remain valid not only for $T$ but also $\mathcal{K}_{i}$ for any $1 \leq i \leq n-1$.

Let us write the action of the time evolution of a monic monomial $p \in \mathcal{P}$ as $T(p)=\sum_{p^{\prime}} A_{p^{\prime}, p} p^{\prime}$, where the sum is taken over monic monomials $p^{\prime} \in \mathcal{P}$. We then define the transposition ${ }^{t} T$ of $T$ by ${ }^{t} T(p)=\sum_{p^{\prime}} A_{p, p^{\prime}} p^{\prime}$.

\section{Proposition 2.7.}

$$
{ }^{t} T=T^{-1}
$$

Proof. In view of Remark 2.5, the inverse $T^{-1}=\mathcal{K}_{1}^{-1}$ is obtained by reversing the horizontal arrows in Fig. 2 and sending the carrier from the right to the left correspondingly in Fig. 3. By using this fact, one can verify the claim. See also Remark 2.13.

Let $\left(\right.$, ) be the inner product such that $\left(p, p^{\prime}\right)=\delta_{p, p^{\prime}}$ for all the monic monomials $p$ and $p^{\prime}$. It is well defined on a subset of $\mathcal{P} \times \mathcal{P}$. Then Proposition 2.7 tells that $(T(r), T(s))=(r, s)$ for $(r, s)$ belonging to the subset. This property leads to a family of $q$-series identities. In fact one has $\sum_{p} A_{p, r} A_{p, s}=\delta_{r s}$ for any monic monomials $r$ and $s$. Pick the monomial $p=\cdots \otimes v_{2} \otimes v_{1} \otimes v_{2} \otimes \cdots$ for instance. Then the left hand side of $(T(p), T(p))=1$, the sum of squared amplitudes, is calculated as

$$
(-q)^{2}+\sum_{k \geq 0}\left(q^{k}\left(1-q^{2}\right)\right)^{2}=1
$$

Similarly for the monomial $p=\cdots \otimes v_{2} \otimes v_{1} \otimes v_{1} \otimes v_{2} \otimes \cdots$, the contributions to $(T(p), T(p))=1$ are grouped into the four cases as in Fig. 4, which add up to 1 . Here the symbols $\bullet$ and $\circ$ stand for a ball $v_{1}$ and an empty box $v_{2}$, respectively. The symbol $\cdots$ represents an array of empty boxes of the specified number. In 
Figure 4. Squared amplitudes for $T(p)$

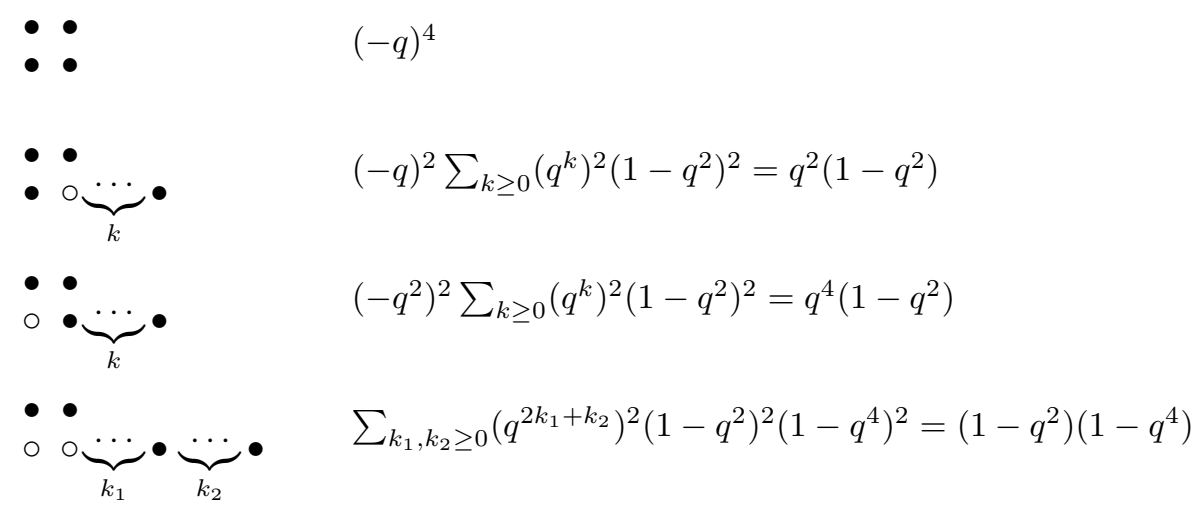

each group, the upper configuration is $p$ and the lower one is a monomial occurring in $T(p)$.

So far we have considered the quadratic form (, ). Now we turn to a linear one. We use the standard notation

$$
\begin{aligned}
& (z)_{m}=(z ; q)_{m}=(1-z)(1-z q) \cdots\left(1-z q^{m-1}\right), \\
& {\left[\begin{array}{c}
m \\
k
\end{array}\right]=\frac{(q)_{m}}{(q)_{k}(q)_{m-k}} .}
\end{aligned}
$$

For $t \leq \min (l, m)$, let $\beta_{m, t, l}$ be the sum of all the amplitudes for $l$ successive vacant boxes to acquire $t$ balls during the passage of a carrier containing $m$ balls. Namely, it is the sum of the amplitudes for Fig. 5 over $1 \leq i_{1}<i_{2}<\cdots<i_{t} \leq l$.

FiguRE $5 . \beta_{m, t, l}$

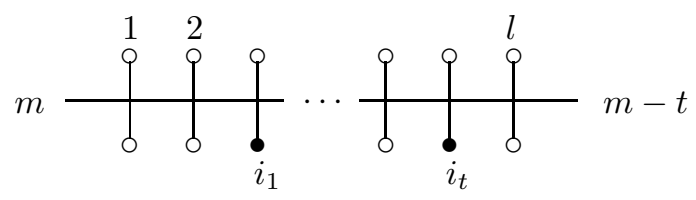

\section{Lemma 2.8.}

$$
\beta_{m, t, l}=q^{(m-t)(l-t)}\left(1-q^{2 m}\right)\left(1-q^{2 m-2}\right) \cdots\left(1-q^{2(m-t+1)}\right)\left[\begin{array}{l}
l \\
t
\end{array}\right] .
$$

Proof. The contribution from Fig. 5 is

$$
\begin{aligned}
& \left(1-q^{2 m}\right)\left(1-q^{2 m-2}\right) \cdots\left(1-q^{2(m-t+1)}\right) \\
& \times q^{m\left(i_{1}-1\right)+(m-1)\left(i_{2}-i_{1}-1\right)+\cdots+(m-t+1)\left(i_{t}-i_{t-1}-1\right)+(m-t)\left(l-i_{t}\right)} .
\end{aligned}
$$

The claim follows by summing this over $1 \leq i_{1}<i_{2}<\cdots<i_{t} \leq l$.

Let $\mathcal{P}_{\text {fin }}$ be the subspace of $\mathcal{P}$ spanned by the superpositions of monomials $\sum_{p} c_{p} p$ in which $\sum_{p} c_{p}$ exists. For instance, monomials are elements of $\mathcal{P}_{\text {fin }}$. Consider the linear function $\mathcal{N}: \mathcal{P}_{\text {fin }} \rightarrow \mathbb{C}$ that takes value 1 on all the monic monomials. 
Proposition 2.9. $T$ preserves $\mathcal{N}$, i.e., $\mathcal{N}(T(p))=\mathcal{N}(p)$ for any $p \in \mathcal{P}_{\text {fin }}$.

For example for $p=\cdots v_{2} \otimes v_{1} \otimes v_{2} \otimes \cdots$, one has

$$
\mathcal{N}(p)=-q+\sum_{k \geq 0} q^{k}\left(1-q^{2}\right)=1 .
$$

For $p=\cdots v_{2} \otimes v_{1} \otimes v_{1} \otimes v_{2} \otimes \cdots$ considered in Fig. 4, one has

$\mathcal{N}(p)=(-q)^{2}-q \sum_{k \geq 0} q^{k}\left(1-q^{2}\right)-q^{2} \sum_{k \geq 0} q^{k}\left(1-q^{2}\right)+\sum_{k_{1}, k_{2} \geq 0} q^{2 k_{1}+k_{2}}\left(1-q^{2}\right)\left(1-q^{4}\right)=1$.

The remainder of this section 2.7 is devoted to a proof of Proposition 2.9. We begin by introducing a map $\Phi_{m}$ for $m \in \mathbb{Z}_{\geq 0}$, which is a slight generalization of $T$. We set $\Phi_{0}=T$. For $m \geq 1, \Phi_{m}$ acts on $\mathcal{P}_{\text {fin }} \backslash\left\{p_{\text {vac }}\right\}$ as follows. Pick any monic monomial $p \in \mathcal{P}_{\text {fin }} \backslash\left\{p_{\text {vac }}\right\}$ and decompose it uniquely as $p=p_{\text {left }} \otimes p_{\text {right }}$, so that $p_{\text {left }}$ is free of balls and the leftmost component of $p_{\text {right }}$ is a ball. Let $p_{\text {right }}^{\prime}$ be the linear combination of the monic monomials generated by the penetration of the carrier initially containing $m$ balls through $p_{\text {right }}$ to the right. See Fig. 6 .

FIGURE $6 . p_{\text {right }}^{\prime}$

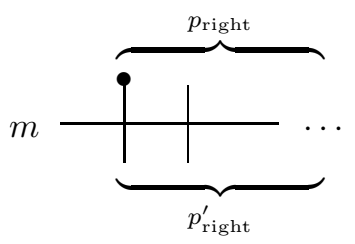

We set $\Phi_{m}(p)=p_{\text {left }} \otimes p_{\text {right }}^{\prime}$ and extend it linearly to the map $\Phi_{m}: \mathcal{P}_{\text {fin }} \backslash\left\{p_{\text {vac }}\right\} \rightarrow$ $\mathcal{P}_{\text {fin }} \backslash\left\{p_{\text {vac }}\right\}$. It is a direct sum of the action $\mathcal{P}_{\text {fin }, N} \rightarrow \mathcal{P}_{\text {fin }, N+m}$ over $N \in \mathbb{Z}_{\geq 1}$, where the notation $\mathcal{P}_{\text {fin, } N}$ is the $n=2$ case of $(2.22)$ restricted to $\mathcal{P}_{\text {fin }}$.

Proposition 2.9 is obvious for $p=p_{\text {vac. }}$. Since $\mathcal{N}$ is linear, the other case follows from the $m=0$ case of

Proposition 2.10. For any monic monomial $p \in \mathcal{P}_{N}$,

$$
\mathcal{N}\left(\Phi_{m}(p)\right)=(1+q)\left(1+q^{2}\right) \cdots\left(1+q^{m}\right)
$$

is valid for any $m \geq 0$ and $N \geq 1$.

The right hand side depends on $m$ but not on $N$, hence it will be denoted by $\alpha_{m}$. Note that $\alpha_{m}=\beta_{m, m, \infty}$.

Proof. We show (2.33) by induction on $N$. For $N=1$, the relevant configurations either accommodate a ball or not just below the initial one. The former contributes $-q^{m+1} \beta_{m, m, \infty}$ to $\mathcal{N}\left(\Phi_{m}(p)\right)$ and the latter does $\beta_{m+1, m+1, \infty}$. The two contributions indeed sum up to $\alpha_{m}$. Assume the claim for $N$. In the monic monomial $p \in \mathcal{P}_{N+1}$, suppose there are $l$ empty boxes between the leftmost ball and its nearest neighbor. The configurations that accommodate $t$ balls in the $l$ boxes are classified into the two cases in Fig. 7. 
Figure 7. Two kinds of contributions to $\mathcal{N}\left(\Phi_{m}(p)\right)$
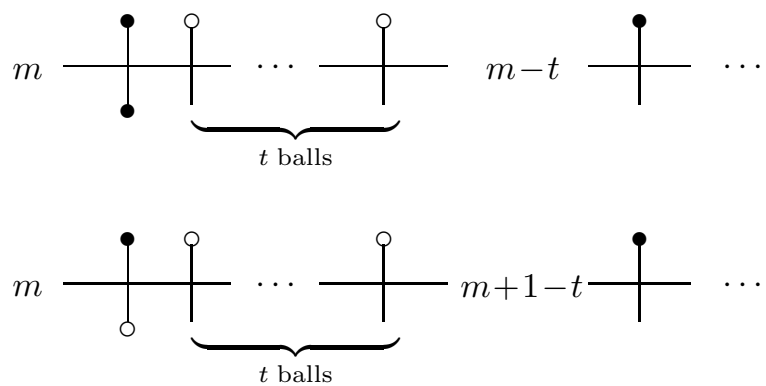

Accordingly we have the recursion relation

$\mathcal{N}\left(\Phi_{m}(p)\right)=-q^{m+1} \sum_{t=0}^{\min (l, m)} \beta_{m, t, l} \mathcal{N}\left(\Phi_{m-t}(\tilde{p})\right)+\sum_{t=0}^{\min (l, m+1)} \beta_{m+1, t, l} \mathcal{N}\left(\Phi_{m+1-t}(\tilde{p})\right)$,

where $\tilde{p} \in \mathcal{P}_{N}$ is the monic monomial obtained by removing the leftmost ball from $p$. Thus we are done if

$$
\alpha_{m}=-q^{m+1} \sum_{t=0}^{\min (l, m)} \beta_{m, t, l} \alpha_{m-t}+\sum_{t=0}^{\min (l, m+1)} \beta_{m+1, t, l} \alpha_{m+1-t}
$$

is shown. This is a corollary of Lemma 2.11 .

Lemma 2.11. Let $l, m \in \mathbb{Z}_{\geq 0}$. Then

$$
\alpha_{m}=\sum_{t=0}^{\min (l, m)} \beta_{m, t, l} \alpha_{m-t} .
$$

Proof. We are to show

$$
1=\sum_{t=0}^{\min (l, m)} q^{(l-t)(m-t)} \frac{(q)_{l}(q)_{m}}{(q)_{t}(q)_{l-t}(q)_{m-t}} .
$$

Since the both sides are symmetric with respect to $l$ and $m$, we assume with no loss of generality that $l \leq m$. Applying the $q$-binomial identity $(z ; q)_{t}=$ $\sum_{s=0}^{t}\left[\begin{array}{c}t \\ s\end{array}\right](-z)^{s} q^{s(s-1) / 2}$, we expand the factor $(q)_{m} /(q)_{m-t}=\left(q^{m-t+1} ; q\right)_{t}$. Then the right hand side becomes

$$
\sum_{t=0}^{l} \sum_{s=0}^{t}(-1)^{s} q^{(m-t)(l-t+s)+s(s+1) / 2} \frac{(q)_{l}}{(q)_{l-t}(q)_{s}(q)_{t-s}} .
$$

By eliminating $t$ by setting $t=s+i$, this is written as

$$
\sum_{i=0}^{l}\left[\begin{array}{l}
l \\
i
\end{array}\right] q^{(m-i)(l-i)} \sum_{s=0}^{l-i}\left[\begin{array}{c}
l-i \\
s
\end{array}\right]\left(-q^{i-l+1}\right)^{s} q^{s(s-1) / 2} .
$$

The $q$-binomial identity tells that the sum over $s$ is equal to $\left(q^{i-l+1} ; q\right)_{l-i}=\delta_{i l}$. 
Remark 2.12. Set $u=\sum_{p} p$, where the sum extends over all the monic monomials in $\mathcal{P}_{N}$ for any $N \geq 0$. Then Proposition 2.7 and Proposition 2.9 tell that $T(u)=u$. Conversely, this property and Proposition 2.7 imply Proposition 2.9 since $\mathcal{N}(p)=$ $(p, u)=(T(p), T(u))=(T(p), u)=\mathcal{N}(T(p))$.

2.8. Bethe ansatz. Consider the commuting family of transfer matrices $T_{m}(z)$ ( $m \in$ $\mathbb{Z}_{>1}$ ) constructed from the fusion $R$ matrix $R^{(m, 1)}(z)(2.3)$. Normalize them so that $T_{m}(z) p_{\text {vac }}=p_{\text {vac }}$. Then the time evolution $T$ of our quantized box-ball system belongs to the family as $T=T_{\infty}(1)$. It therefore shares the eigenvectors with the simplest one $T_{1}(z)$, which corresponds to the well known six vertex model [Bax]. A slight peculiarity here is that we work on $\mathcal{P}$, which implies an infinite system from the onset under a fixed boundary condition. The Bethe ansatz result is adapted to such a circumstance as follows:

$$
\begin{aligned}
& T_{m}(z)\left|\xi_{1}, \ldots, \xi_{N}\right\rangle_{B}=\lambda_{m}\left(z, \xi_{1}\right) \cdots \lambda_{m}\left(z, \xi_{N}\right)\left|\xi_{1}, \ldots, \xi_{N}\right\rangle_{B}, \\
& \left|\xi_{1}, \ldots, \xi_{N}\right\rangle_{B}=\sum_{i_{1}<\cdots<i_{N}} C_{i_{1}, \ldots, i_{N}}\left(\xi_{1}, \ldots, \xi_{N}\right)\left|i_{1}, \ldots, i_{N}\right\rangle, \\
& C_{i_{1}, \ldots, i_{N}}\left(\xi_{1}, \ldots, \xi_{N}\right)=\sum_{P \in \mathfrak{S}_{N}} \operatorname{sign}(P)\left(\prod_{j<k} A_{P_{j}, P_{k}}\right) \xi_{P_{1}}^{i_{1}} \cdots \xi_{P_{N}}^{i_{N}}, \\
& A_{j, k}=q \eta_{j}-q^{-1} \eta_{k}, \quad \eta_{i}=\frac{1-q \xi_{i}}{\xi_{i}-q}, \quad \lambda_{m}\left(z, \xi_{i}\right)=\frac{q^{m}+\eta_{i} z}{1+q^{m} \eta_{i} z},
\end{aligned}
$$

where $N$ is an arbitrary nonnegative integer, $|\cdots\rangle_{B} \in \mathcal{P}_{N}$ is the joint eigenvector of Bethe, and $\left|i_{1}, \ldots, i_{N}\right\rangle$ is the monic monomial describing the ball configuration at positions $i_{1}, \ldots, i_{N}$. The sum over $P$ runs over the symmetric group $\mathfrak{S}_{N}$, and $\operatorname{sign}(P)= \pm 1$ denotes the signature of $P$. The above result holds for $q \in \mathbb{R}$ such that $-1<q<1$ and $z \in \mathbb{C}$ such that $|z q|<1$. The parameters $\xi_{1}, \ldots, \xi_{N}$ should be all distinct for the Bethe vector not to vanish. They are to be taken from $\exp (\sqrt{-1} \mathbb{R})$ to match the condition (ii) in (2.21), but otherwise arbitrary free from the Bethe equation. One sees that $\lambda_{m}\left(z, \xi_{i}\right)$ tends to $\eta_{i} z$ in the limit $q^{m} \rightarrow 0$ in agreement with (2.27) with $n=2$. The one particle eigenvalue $\lambda_{m}(z)=\lambda_{m}\left(z, \xi_{i}\right)$ satisfies the degenerate $T$ system $\lambda_{m}(z q) \lambda_{m}\left(z q^{-1}\right)=\lambda_{m+1}(z) \lambda_{m-1}(z)$. Except the obvious $N=1$ case, it is not known to us whether the property $T(u)=u$ in Remark 2.12 can be deduced from the Bethe ansatz result quoted here.

Remark 2.13. In terms of $T_{m}(z)$ considered here and its transposition defined similarly to section 2.7 , Proposition 2.7 is the $m \rightarrow \infty$ case of ${ }^{t} T_{m}\left(z^{-1}\right)=T_{m}(z)^{-1}$ derivable from the inversion relation (2.7).

\section{3. $\boldsymbol{D}_{\boldsymbol{n}}^{(1)}$ CASE}

3.1. $\boldsymbol{R}$ matrix $\boldsymbol{R}(\boldsymbol{z})$. Let $J=\{1,2, \cdots, n,-n,-n+1, \cdots-1\}$ be the set equipped with an order $1 \prec 2 \prec \cdots \prec n \prec-n \prec \cdots \prec-2 \prec-1$. In the following, elements of $2 n \times 2 n$ matrices with indices from $J$ are arranged in the increasing order with respect to $\prec$ from the top left. We use the notation

$$
\xi=q^{2 n-2}, \quad \bar{i}= \begin{cases}i & i>0, \\ i+2 n & i<0 .\end{cases}
$$


Let $V=\oplus_{\mu \in J} \mathbb{C} v_{\mu}$ be the vector representation of $U_{q}\left(D_{n}^{(1)}\right)$. The $R$ matrix $R(z) \in$ $\operatorname{End}(V \otimes V)$ was obtained in [B, J]. Here we start with the following convention:

$$
\begin{aligned}
R(z) & =a(z) \sum_{k} E_{k k} \otimes E_{k k}+b(z) \sum_{j \neq k} E_{j j} \otimes E_{k k}+c(z)\left(z \sum_{j \prec k}+\sum_{j \succ k}\right) E_{k j} \otimes E_{j k} \\
& +(z-1)(1-q) \sum_{j, k} f_{j k}(z) E_{j k} \otimes E_{-j-k},
\end{aligned}
$$

where the sums extend over $J$ and $E_{i j} v_{k}=\delta_{j k} v_{i}$.

$$
\begin{aligned}
& a(z)=\left(1-q^{2} z\right)(1-\xi z), b(z)=q(1-z)(1-\xi z), c(z)=\left(1-q^{2}\right)(1-\xi z), \\
& f_{j k}(z)= \begin{cases}q+\xi z & j=k, \\
(1+q)(-1)^{j+k} q^{\bar{k}-\bar{j}} & j \prec k, \\
(1+q)(-1)^{j+k} q^{\bar{k}-\bar{j}} \xi z & j \succ k .\end{cases}
\end{aligned}
$$

The $R$ matrix satisfies the Yang-Baxter equation.

We denote by $\sigma$ the automorphism of $V$ acting as $\sigma v_{ \pm 1}=v_{\mp 1}, \sigma v_{ \pm n}=v_{\mp n}$, and $\sigma v_{\mu}=v_{\mu}$ for $\mu \neq \pm 1, \pm n$.

3.2. Fusion $\boldsymbol{R}$ matrix and its limit. As the $A_{n-1}^{(1)}$ case, we set $V_{1}=V$ and realize the space $V_{m}$ of the $m$ fold $q$-symmetric tensors as the quotient $V^{\otimes m} / A$, where $A=\sum_{j} V^{\otimes j} \otimes \operatorname{Im} P R\left(q^{-2}\right) \otimes V^{\otimes m-2-j}$. The basis of $\operatorname{Im} P R\left(q^{-2}\right)$ can be taken as

$$
\begin{aligned}
& v_{i} \otimes v_{j}-q v_{j} \otimes v_{i}, \text { for } i \prec j, i \neq \pm j, \\
& v_{1} \otimes v_{-1}-q^{2} v_{-1} \otimes v_{1}, \quad v_{n} \otimes v_{-n}-v_{-n} \otimes v_{n}, \\
& v_{j} \otimes v_{-j}-v_{-j} \otimes v_{j}-q v_{-j-1} \otimes v_{j+1}+q^{-1} v_{j+1} \otimes v_{-j-1}, \text { for } 1 \leq j \leq n-1 .
\end{aligned}
$$

A vector of the form $v_{i_{1}} \otimes v_{i_{2}} \otimes \cdots \otimes v_{i_{m}}$ is called normal ordered if $-1 \succeq i_{1} \succeq$ $\cdots \succeq i_{m} \succeq 1$ and the sequence $i_{1}, \ldots, i_{m}$ does not contain the letters $n$ and $-n$ simultaneously. The set of normal ordered vectors $v_{i_{1}} \otimes v_{i_{2}} \otimes \cdots \otimes v_{i_{m}} \bmod A$ form the basis of $V_{m}$. We label them as $x=\left[x_{1}, \ldots, x_{n}, x_{-n}, \ldots, x_{-1}\right]$, where $x_{i} \in \mathbb{Z}_{\geq 0}$ is the number of the letter $i$ in the sequence $i_{1}, \ldots, i_{m}$. Thus $x_{1}+\cdots+x_{-1}=m$ and $x_{n} x_{-n}=0$ hold in accordance with the label in [KKM]. In $V^{\otimes m}$ normal ordering is done according to the local rule $\bmod \operatorname{Im} P R\left(q^{-2}\right)$ :

$$
\begin{aligned}
& v_{1} \otimes v_{-1}=q^{2} v_{-1} \otimes v_{1}, \quad v_{i} \otimes v_{j}=q v_{j} \otimes v_{i} \quad i \prec j, i \neq \pm j, \\
& v_{j} \otimes v_{-j}=q^{2} v_{-j} \otimes v_{j}-\left(1-q^{2}\right) \sum_{i=1}^{j-1}(-q)^{j-i} v_{-i} \otimes v_{i} \quad 2 \leq j \leq n-1, \\
& v_{n} \otimes v_{-n}=v_{-n} \otimes v_{n}=-\sum_{i=1}^{n-1}(-q)^{n-i} v_{-i} \otimes v_{i} .
\end{aligned}
$$

Then the fusion $R$ matrix $R^{(m, 1)}(z)$ is the restriction of the operator (2.2) to $\operatorname{End}\left(V_{m} \otimes V\right)$. For $x \in V_{m}$ and $\mu \in J$ we set

$$
R^{(m, 1)}(z)\left(x \otimes v_{\mu}\right)=\sum_{\nu \in J, y \in V_{m}} w_{\mu \nu}[x \mid y]\left(y \otimes v_{\nu}\right) .
$$


Due to the weight conservation the matrix element $w_{\mu \nu}[x \mid y]$ is zero unless

$$
\operatorname{wt}(x)+\operatorname{wt}\left(v_{\mu}\right)=\operatorname{wt}(y)+\operatorname{wt}\left(v_{\nu}\right),
$$

where the weights may be regarded as elements in $\mathbb{Z}^{n}$ by

$$
\begin{aligned}
& \operatorname{wt}\left(\left[x_{1}, \ldots, x_{n}, x_{-n}, \ldots, x_{-1}\right]\right)=\left(x_{1}-x_{-1}, \ldots, x_{n}-x_{-n}\right), \\
& \operatorname{wt}\left(v_{\mu}\right)=(0 \ldots, 0, \stackrel{|\mu| \text { th }}{ \pm 1}, 0, \ldots, 0) \text { for } \pm \mu>0 .
\end{aligned}
$$

Leaving the calculation of $w_{\mu \nu}[x \mid y]$ in general case aside, we present the result for the limit

$$
W_{\mu \nu}[x \mid y]:=\lim _{x_{-n} \rightarrow \infty} w_{\mu \nu}[x \mid y] .
$$

Note that one necessarily has $x_{n}=y_{n}=0$ by the weight reason. Therefore $x$ appearing in $W_{\mu \nu}[x \mid y]$ is to be understood as the array $\left(x_{1}, \ldots, x_{n-1}, x_{-n+1}, \ldots, x_{-1}\right)$ that does not contain the $\pm n$ components, and the same applies to $y$ as well. For positive integers $j$ and $k$ such that $j \leq k$ we use the symbols

$$
x_{j, k}=x_{j}+x_{j+1}+\cdots+x_{k}, \quad x_{-j,-k}=x_{-j}+x_{-j-1}+\cdots+x_{-k} .
$$

They are to be understood as zero for $j>k$. Derivation of $W_{\mu \nu}[x \mid y]$ is outlined in Appendix A. We summarize the result in

Proposition 3.1. Suppose $j, k, l \in\{1,2, \ldots, n-1\}$. The nonzero matrix elements $W_{\mu \nu}[x \mid y]$ are exhausted by the following list:

$$
\begin{aligned}
& W_{ \pm j, \pm j}[x \mid x]=-z q^{x_{j}+x_{-j}+1}, \\
& W_{j<k}[x \mid x-(-j)+(-k)]=(-1)^{j+k} z\left(1-q^{2 x_{-j}}\right) q^{k-j+x_{j}+x_{-j-1,-k+1}}, \\
& W_{j>k}[x \mid x+(j)-(k)]=z\left(1-q^{2 x_{k}}\right) q^{x_{k+1, j-1}+x_{-k}}, \\
& W_{j, k}[x \mid x-(l)-(-l)+(j)+(-k)]_{l<\min (j, k)} \\
& =(-1)^{k+l+1} z\left(1-q^{2 x_{l}}\right)\left(1-q^{2 x_{-l}}\right) q^{k-l-1+x_{l+1, j-1}+x_{-l-1,-k+1}}, \\
& W_{-j>-k}[x \mid x+(-j)-(-k)]=z\left(1-q^{2 x_{-k}}\right) q^{x_{j}+x_{-j-1,-k+1}}, \\
& W_{-j<-k}[x \mid x+(k)-(j)]=(-1)^{j+k} z\left(1-q^{2 x_{j}}\right) q^{j-k+x_{k+1, j-1}+x_{-k}}, \\
& W_{-j,-k}[x \mid x+(l)+(-l)-(j)-(-k)]_{l<\min (j, k)} \\
& \quad=(-1)^{j+l+1} z\left(1-q^{2 x_{j}}\right)\left(1-q^{2 x_{-k}}\right) q^{j-l-1+x_{l+1, j-1}+x_{-l-1,-k+1}}, \\
& W_{-j, k}[x \mid x-(j)+(-k)]=(-1)^{j+k} z^{2}\left(1-q^{2 x_{j}}\right) q^{j+k-2+x_{1, j-1}+x_{-1,-k+1}}, \\
& W_{j,-k}[x \mid x+(j)-(-k)]=\left(1-q^{2 x_{-k}}\right) q^{x_{1, j-1}+x_{-1,-k+1}}, \\
& \quad W_{n, k}[x \mid x-(-n)+(-k)]=(-1)^{n+k} z^{2} q^{n+k-2+x_{1, n-1}+x_{-1,-k+1}}, \\
& W_{n,-k}[x \mid x-(-n)+(k)]=(-1)^{n+k} z q^{n-k+x_{k+1, n-1}+x_{-k}}, \\
& W_{n,-k}[x \mid x+(l)+(-l)-(-n)-(-k)]_{l<k} \\
& \quad=(-1)^{n+l+1} z\left(1-q^{2 x_{-k}}\right) q^{n-l-1+x_{l+1, n-1}+x_{-l-1,-k+1}},
\end{aligned}
$$




$$
\begin{aligned}
& W_{-n, k}[x \mid x+(-n)-(k)]=z\left(1-q^{2 x_{k}}\right) q^{x_{k+1, n-1}+x_{-k}}, \\
& W_{-n, k}[x \mid x-(l)-(-l)+(-n)+(-k)]_{l<k} \\
& =(-1)^{k+l+1} z\left(1-q^{2 x_{l}}\right)\left(1-q^{2 x_{-l}}\right) q^{k-l-1+x_{l+1, n-1}+x_{-l-1,-k+1}}, \\
& W_{-n,-k}[x \mid x+(-n)-(-k)]=\left(1-q^{2 x_{-k}}\right) q^{x_{1, n-1}+x_{-1,-k+1}} \text {, } \\
& W_{j, n}[x \mid x-(-j)+(-n)]=(-1)^{j+n} z\left(1-q^{2 x_{-j}}\right) q^{n-j+x_{j}+x_{-j-1,-n+1}}, \\
& W_{j, n}[x \mid x-(l)-(-l)+(j)+(-n)]_{l<j} \\
& =(-1)^{l+n+1} z\left(1-q^{2 x_{l}}\right)\left(1-q^{2 x_{-l}}\right) q^{n-l-1+x_{l+1, j-1}+x_{-l-1,-n+1}}, \\
& W_{-j, n}[x \mid x-(j)+(-n)]=(-1)^{j+n} z^{2}\left(1-q^{2 x_{j}}\right) q^{n+j-2+x_{1, j-1}+x_{-1,-n+1}} \text {, } \\
& W_{j,-n}[x \mid x+(j)-(-n)]=q^{x_{1, j-1}+x_{-1,-n+1}}, \\
& W_{-j,-n}[x \mid x-(-n)+(-j)]=z q^{x_{j}+x_{-j-1,-n+1}} \text {, } \\
& W_{-j,-n}[x \mid x-(j)-(-n)+(l)+(-l)]_{l<j} \\
& =(-1)^{j+l+1} z\left(1-q^{2 x_{j}}\right) q^{j-l-1+x_{l+1, j-1}+x_{-l-1,-n+1}} \text {, } \\
& W_{n, n}[x \mid x]=z^{2} q^{2 n-2+x_{1, n-1}+x_{-1,-n+1}} \\
& W_{-n,-n}[x \mid x]=q^{x_{1, n-1}+x_{-1,-n+1}}, \\
& W_{n,-n}[x \mid x-2(-n)+(l)+(-l)]=(-1)^{n+l+1} z q^{n-l-1+x_{l+1, n-1}+x_{-l-1,-n+1}} \text {, } \\
& W_{-n, n}[x \mid x+2(-n)-(l)-(-l)] \\
& =(-1)^{n+l+1} z\left(1-q^{2 x_{l}}\right)\left(1-q^{2 x_{-l}}\right) q^{n-l-1+x_{l+1, n-1}+x_{-l-1,-n+1}} .
\end{aligned}
$$

Here the notation $y=x+(l)+(-l)-(j)-(-k)$ for example means that $y$ is obtained from $x$ by setting $x_{l} \rightarrow x_{l}+1, x_{-l} \rightarrow x_{-l}+1, x_{j} \rightarrow x_{j}-1, x_{-k} \rightarrow x_{-k}-1$. Since $x_{-n}$ becomes irrelevant in the limit $(3.8),(-n)$ in the argument of $W_{\mu \nu}$ may just be dropped. It has been included in the above formulas as a reminder of the conservation of the number of components. The matrix elements of the form $W_{\mu \nu}[x \mid x-(\lambda) \pm \cdots]$ with any $\lambda \in\{ \pm 1, \ldots, \pm(n-1)\}$ contain the factor $1-q^{2 x_{\lambda}}$ as they should.

3.3. $\boldsymbol{L}$ operator $\boldsymbol{L}(\boldsymbol{z})$. We consider the Weyl algebra generated by $P_{\mu}^{ \pm 1}, Q_{\mu}^{ \pm 1}$ with $\mu \in J \backslash\{ \pm n\}$ under the same relation as (2.10). The subalgebra of the Weyl algebra generated by $P_{\mu}, Q_{\mu}$ and $R_{\mu}=Q_{\mu}^{-1}\left(1-a_{\mu} P_{\mu}^{2}\right)$ with $\mu \in J \backslash\{ \pm n\}$ will again be denoted by $\mathcal{A}$, where $a_{\mu}$ is a parameter. We define the $L$ operator $L(z)=\left(L_{\mu \nu}(z)\right)_{\mu, \nu \in J} \in \mathcal{A} \otimes \operatorname{End}(V)$ so that $L_{\mu \nu}(z) \in \mathcal{A}$ with $\forall a_{\mu}=1$ becomes the operator version of $W_{\nu \mu}[x \mid y]$ in Proposition 3.1. See (3.15). To present it explicitly, we assume $1 \leq j, k, l \leq n-1$ in this subsection. We set $P_{\mu}^{\prime}=-q a_{\mu} P_{\mu}$ and use the symbols

$$
\begin{aligned}
& P_{j, k}=P_{j} P_{j+1} \cdots P_{k}, \quad P_{-j,-k}=P_{-j} P_{-j-1} \cdots P_{-k}, \\
& P_{j, k}^{\prime}=P_{j}^{\prime} P_{j+1}^{\prime} \cdots P_{k}^{\prime}, \quad P_{-j,-k}^{\prime}=P_{-j}^{\prime} P_{-j-1}^{\prime} \cdots P_{-k}^{\prime}
\end{aligned}
$$


for $j \leq k$. For $j>k$ they should be understood as 1 . Then $L_{\mu \nu}(z) \in \mathcal{A}$ reads as follows:

$$
\begin{aligned}
& L_{j j}(z)=z P_{j}^{\prime} P_{-j}+z \sum_{l=1}^{j-1} R_{-l} P_{-l-1,-j+1}^{\prime} Q_{-j} R_{l} P_{l+1, j-1} Q_{j}, \\
& L_{-j,-j}(z)=z P_{j} P_{-j}^{\prime}+z \sum_{l=1}^{j-1} Q_{-l} P_{-l-1,-j+1} R_{-j} Q_{l} P_{l+1, j-1}^{\prime} R_{j}, \\
& L_{k>j}(z)=z R_{-j} P_{-j-1,-k+1}^{\prime} Q_{-k} P_{j}^{\prime}+z \sum_{l=1}^{j-1} R_{-l} P_{-l-1,-k+1}^{\prime} Q_{-k} R_{l} P_{l+1, j-1} Q_{j}, \\
& L_{k<j}(z)=z P_{-k} R_{k} P_{k+1, j-1} Q_{j}+z \sum_{l=1}^{k-1} R_{-l} P_{-l-1,-k+1}^{\prime} Q_{-k} R_{l} P_{l+1, j-1} Q_{j}, \\
& L_{-k<-j}(z)=z Q_{-j} P_{-j-1,-k+1} R_{-k} P_{j}+z \sum_{l=1}^{j-1} Q_{-l} P_{-l-1,-k+1} R_{-k} Q_{l} P_{l+1, j-1}^{\prime} R_{j}, \\
& L_{-k>-j}(z)=z P_{-k}^{\prime} Q_{k} P_{k+1, j-1}^{\prime} R_{j}+z \sum_{l=1}^{k-1} Q_{-l} P_{-l-1,-k+1} R_{-k} Q_{l} P_{l+1, j-1}^{\prime} R_{j}, \\
& L_{k,-j}(z)=z^{2} P_{-1,-k+1}^{\prime} Q_{-k} P_{1, j-1}^{\prime} R_{j}, \\
& L_{-k, j}(z)=P_{-1,-k+1} R_{-k} P_{1, j-1} Q_{j},
\end{aligned}
$$

$$
\begin{aligned}
& L_{k, n}(z)=z^{2} P_{-1,-k+1}^{\prime} Q_{-k} P_{1, n-1}^{\prime}, \\
& L_{-k, n}(z)=z P_{-k}^{\prime} Q_{k} P_{k+1, n-1}^{\prime}+z \sum_{l=1}^{k-1} Q_{-l} P_{-l-1,-k+1} R_{-k} Q_{l} P_{l+1, n-1}^{\prime}, \\
& L_{k,-n}(z)=z P_{-k} R_{k} P_{k+1, n-1}+z \sum_{l=1}^{k-1} R_{-l} P_{-l-1,-k+1}^{\prime} Q_{-k} R_{l} P_{l+1, n-1}, \\
& L_{-k,-n}(z)=P_{-1,-k+1} R_{-k} P_{1, n-1}, \\
& L_{n, j}(z)=z R_{-j} P_{-j-1,-n+1}^{\prime} P_{j}^{\prime}+z \sum_{l=1}^{j-1} R_{-l} P_{-l-1,-n+1}^{\prime} R_{l} P_{l+1, j-1} Q_{j}, \\
& L_{n,-j}(z)=z^{2} P_{-1,-n+1}^{\prime} P_{1, j-1}^{\prime} R_{j}, \\
& L_{-n, j}(z)=P_{-1,-n+1} P_{1, j-1} Q_{j}, \\
& L_{-n,-j}(z)=z P_{j} Q_{-j} P_{-j-1,-n+1}+z \sum_{l=1}^{j-1} Q_{-l} P_{-l-1,-n+1} Q_{l} P_{l+1, j-1}^{\prime} R_{j},
\end{aligned}
$$




$$
\begin{aligned}
& L_{n, n}(z)=z^{2} P_{1, n-1}^{\prime} P_{-1,-n+1}^{\prime} \\
& L_{-n, n}(z)=z \sum_{l=1}^{n-1} Q_{l} P_{-l-1,-n+1} Q_{-l} P_{l+1, n-1}^{\prime}, \\
& L_{n,-n}(z)=z \sum_{l=1}^{n-1} R_{-l} P_{-l-1,-n+1}^{\prime} R_{l} P_{l+1, n-1}, \\
& L_{-n,-n}(z)=P_{1, n-1} P_{-1,-n+1} .
\end{aligned}
$$

In these formulas, the operators $P_{\mu}, Q_{\mu}, R_{\mu}$ and $P_{\mu}^{\prime}$ appearing in a single summand always have distinct indices hence their ordering does not matter.

3.4. Factorization of $\boldsymbol{L}(\boldsymbol{z})$. For $\mu \in J \backslash\{ \pm n\}$, let $K_{\mu}=\left(\left(K_{\mu}\right)_{\lambda, \nu}\right)_{\lambda, \nu \in J} \in$ $\mathcal{A} \otimes \operatorname{End}(V)$ be the operator having the elements

$$
\begin{aligned}
& \left(K_{\mu}\right)_{-n, \mu}=\left(K_{\mu}\right)_{-\mu, n}=Q_{\mu}, \\
& \left(K_{\mu}\right)_{\mu,-n}=\left(K_{\mu}\right)_{n,-\mu}=R_{\mu}, \\
& \left(K_{\mu}\right)_{-n,-n}=\left(K_{\mu}\right)_{-\mu,-\mu}=P_{\mu}, \\
& \left(K_{\mu}\right)_{\mu, \mu}=\left(K_{\mu}\right)_{n, n}=P_{\mu}^{\prime}, \\
& \left(K_{\mu}\right)_{\nu, \nu}=1 \quad \nu \neq \pm \mu, \pm n .
\end{aligned}
$$

All the other elements are zero. Here $R_{\mu}=Q_{\mu}^{-1}\left(1-a_{\mu} P_{\mu}^{2}\right)$ and $P_{\mu}^{\prime}=-q a_{\mu} P_{\mu}$ as in section 3.3. We also introduce $S_{\mu}, \bar{S}_{\mu} \in \mathcal{A} \otimes \operatorname{End}(V)$ for $\mu=0, \ldots, n$ as follows. First we specify $S_{1}, \ldots, S_{n-1}$ by

$$
\begin{aligned}
& \left(S_{\mu}\right)_{\mu, \mu}=\left(S_{\mu}\right)_{-\mu-1,-\mu-1}=Q_{\mu}, \\
& \left(S_{\mu}\right)_{\mu+1, \mu+1}=\left(S_{\mu}\right)_{-\mu,-\mu}=R_{\mu}, \\
& \left(S_{\mu}\right)_{\mu, \mu+1}=\left(S_{\mu}\right)_{-\mu-1,-\mu}=P_{\mu}, \\
& \left(S_{\mu}\right)_{\mu+1, \mu}=\left(S_{\mu}\right)_{-\mu,-\mu-1}=P_{\mu}^{\prime}, \\
& \left(S_{\mu}\right)_{\nu, \nu}=1 \quad \nu \neq \pm \mu, \pm(\mu+1),
\end{aligned}
$$

where the other elements are zero. Then $\bar{S}_{\mu} \in \mathcal{A} \otimes \operatorname{End}(V)$ with $1 \leq \mu \leq n-1$ is obtained from $S_{\mu}$ by replacing $P_{\mu}, Q_{\mu}, R_{\mu}$ and $P_{\mu}^{\prime}$ with $P_{-\mu}, Q_{-\mu}, R_{-\mu}=Q_{-\mu}^{-1}(1-$ $\left.a_{-\mu} P_{-\mu}^{2}\right)$ and $P_{-\mu}^{\prime}=-q a_{-\mu} P_{-\mu}$, respectively. Finally the remaining ones are determined by

$$
S_{0}=\sigma S_{1} \sigma, \quad S_{n}=\sigma S_{n-1} \sigma, \quad \bar{S}_{0}=\sigma \bar{S}_{1} \sigma, \quad \bar{S}_{n}=\sigma \bar{S}_{n-1} \sigma
$$

where $\sigma=\sigma^{-1}$ is defined in the end of section 3.1.

The operators $K_{\mu}$ and $S_{\nu}, \bar{S}_{\nu}$ are connected via a gauge transformation analogous to (2.17). To explain it we prepare the Weyl group operators $\sigma_{0}, \ldots, \sigma_{n} \in \operatorname{End}(V)$ which act as identity except

$$
\begin{aligned}
& \sigma_{0}: v_{1} \leftrightarrow v_{-2}, \quad v_{-1} \leftrightarrow v_{2}, \\
& \sigma_{i}: v_{i} \leftrightarrow v_{i+1}, \quad v_{-i} \leftrightarrow v_{-i-1} \quad 1 \leq i \leq n-1, \\
& \sigma_{n}: v_{n-1} \leftrightarrow v_{-n}, \quad v_{-n+1} \leftrightarrow v_{n} .
\end{aligned}
$$

In terms of the sequences

$$
\begin{aligned}
& \left(i_{2 n-2}, \ldots, i_{2}, i_{1}\right)=(n, n-2, n-3, \ldots, 2,0,1,2, \ldots, n-2, n), \\
& \left(\mu_{2 n-2}, \ldots, \mu_{2}, \mu_{1}\right)=(-n+1, \ldots,-2,-1,1,2, \ldots, n-1),
\end{aligned}
$$


the gauge transformation is given by

$$
K_{\mu_{k}}= \begin{cases}\sigma_{i_{1}} \cdots \sigma_{i_{k}} S_{i_{k}} \sigma_{i_{k-1}} \cdots \sigma_{i_{1}} & 1 \leq k \leq n-1, \\ \sigma_{i_{1}} \cdots \sigma_{i_{k}} \bar{S}_{i_{k}} \sigma_{i_{k-1}} \cdots \sigma_{i_{1}} & n \leq k \leq 2 n-2 .\end{cases}
$$

We note the relations

$$
\begin{aligned}
& \sigma=\sigma_{i_{1}} \cdots \sigma_{i_{2 n-2}}, \\
& \sigma S_{i} \sigma=S_{i}, \quad \sigma \bar{S}_{i} \sigma=\bar{S}_{i} \quad 1 \leq i \leq n-1 .
\end{aligned}
$$

Define the diagonal matrices

$$
\begin{aligned}
& d(z)=z \operatorname{diag}(z^{-1} \overbrace{1, \ldots, 1}^{2 n-2}, z), \\
& D(z)=\sigma_{i_{1}} \cdots \sigma_{i_{n-1}} d(z) \sigma_{i_{n-1}} \cdots \sigma_{i_{1}}=z \operatorname{diag}(\overbrace{1, \ldots, 1}^{n-1}, z, z^{-1}, \overbrace{1, \ldots, 1}^{n-1}) .
\end{aligned}
$$

Proposition 3.2. The L operator in section 3.3 is factorized as

$$
L(z)=K_{-n+1} \cdots K_{-1} D(z) K_{1} \cdots K_{n-1} .
$$

Equivalently it is also expressed as

$$
\begin{aligned}
L(z) & =\sigma \bar{S}_{i_{2 n-2}} \cdots \bar{S}_{i_{n}} d(z) S_{i_{n-1}} \cdots S_{i_{1}} \\
& =\bar{S}_{n-1} \bar{S}_{n-2} \cdots \bar{S}_{2} \bar{S}_{1} \sigma d(z) S_{1} S_{2} \cdots S_{n-2} S_{n} .
\end{aligned}
$$

The equivalence of the first and the second expressions is due to (3.12) and (3.14). The second one and the third are connected by (3.11) and (3.13). The first expression is proved in Appendix B.

Proposition 3.3. The $L$ operator and the $R$ matrix (3.2) satisfy the same $R L L$ relation as in Proposition 2.1.

Proposition 3.3 is a corollary of Proposition 3.2 and

Lemma 3.4.

$$
\begin{aligned}
& R\left(z_{2} / z_{1}\right)\left(\sigma d\left(z_{1}\right) \otimes \sigma d\left(z_{2}\right)\right)=\left(\sigma d\left(z_{1}\right) \otimes \sigma d\left(z_{2}\right)\right) R\left(z_{2} / z_{1}\right), \\
& R(z) \stackrel{2}{S_{\mu}} \stackrel{1}{S}_{\mu}=\stackrel{1}{S}_{\mu} \stackrel{2}{S}_{\mu} R(z), \quad 1 \leq \mu \leq n \\
& R(z) \stackrel{2}{\bar{S}_{\mu}}{\stackrel{1}{S_{\mu}}}_{=}=\bar{S}_{\mu} \stackrel{2}{S}_{\mu} R(z), \quad 1 \leq \mu \leq n .
\end{aligned}
$$

Proof. The first relation is straightforward to check. Next consider the second relation with $1 \leq \mu \leq n-1$. Comparing the $R$ matrices (2.1) and (3.2), we find that the contributions proportional to $a(z), b(z)$ and $c(z)$ on the both sides are equal due to Lemma 2.4 for $A_{n-1}^{(1)}$ case. Thus we are to show the equality with $R(z)$ replaced with $\sum_{j, k} f_{j k}(z) E_{j k} \otimes E_{-j-k}$. It is easily checked at $z=0$ and $z=\xi^{-1}$ for example, which suffices since $f_{j k}(z)$ is linear in $z$. Then the second relation with $\mu=n$ follows from $\mu=n-1$ case by using $S_{n}=(\sigma d(z))^{-1} S_{n-1} \sigma d(z)$. The third relation can be shown similarly.

As Remark 2.5, if $a_{\mu}=1$, the property $K_{\mu}^{2}=\mathbb{1}_{2 n}$ holds for any $\mu \in J \backslash\{ \pm n\}$. 
3.5. Quantized $\boldsymbol{D}_{\boldsymbol{n}}^{(1)}$ automaton. Here we set up the quantized $D_{n}^{(1)}$ automaton. It is a system of particles and antiparticles on one dimensional lattice whose dynamics is governed by the $L$ operator constructed in section 3.3. In the limit $q \rightarrow 0$, the dynamics become deterministic and the system reduces to the $D_{n}^{(1)}$ automaton [HKT3, HKT1]. Since our results are parallel with those in subsections $2.4-2.7$, we shall only give a brief sketch and omit the details.

The space of states $\mathcal{P}$ is given by $(2.21)$, where $V$ is now understood as the $2 n$ dimensional vector representation $V=\mathbb{C} v_{1} \oplus \cdots \oplus \mathbb{C} v_{-1}$. The condition (ii) remains the same while the condition (i) is replaced by $\sum_{k \in \mathbb{Z}}\left|j_{k}+n\right|<\infty$.

Monomials $\cdots \otimes v_{j_{-1}} \otimes v_{j_{0}} \otimes v_{j_{1}} \otimes \cdots$ can be classified according to the numbers $w_{1}, \ldots, w_{n}, w_{-n+1}, \ldots, w_{-1}$ of occurrence of the letters $1, \ldots, n,-n+1, \ldots,-1$ in the set $\left\{j_{k}\right\}$. Consequently one has the direct sum decomposition $\mathcal{P}=\oplus \mathcal{P}_{w_{1}, \ldots, w_{-1}}$ analogous to $(2.22)$, where $\mathcal{P}_{0, \ldots, 0}=\mathbb{C} p_{\text {vac }}$ with $p_{\text {vac }}=\cdots \otimes v_{-n} \otimes v_{-n} \otimes \cdots$.

The local states $v_{j_{k}} \in V$ is regarded as the $k$ th box containing a particle of color $j_{k}$ if $j_{k} \in\{ \pm 1, \ldots, \pm(n-1)\}$. Particles having colors with opposite signs are regarded as antiparticles of the other. The case $j_{k}=-n$ is interpreted as an empty box, while $j_{k}=n$ represents a bound state of a particle and an antiparticle.

To formulate the time evolution, we assume $\forall a_{\mu}=1$ from now on, and consider the space of the quantum carrier, namely, the $\mathcal{A}$ module $\mathcal{M}$ defined similarly to (2.23). The difference now is that we need $2 n-2$ coordinates and to set $\mathcal{M}=\oplus \mathbb{C}\left[m_{1}, \ldots, m_{n-1}, m_{-n+1}, \ldots, m_{-1}\right]$. Then the actions of $P_{\mu}, Q_{\mu}, R_{\mu}$ and $P_{\mu}^{\prime}=-q P_{\mu}$ are again given by $(2.23)$ by simply extending the index $i$ to $\mu= \pm 1, \ldots, \pm(n-1)$. By construction we have

$$
L(z)\left(x \otimes v_{\mu}\right)=\sum_{\nu \in J, y \in \mathcal{M}} W_{\mu \nu}[x \mid y]\left(y \otimes v_{\nu}\right)
$$

for $x \in \mathcal{M}$. Here the sum over $y$ is taken under the constraint (3.6), where the weight wt should now be understood as (3.7) without the $n$th component.

The time evolution $T(z): \mathcal{P} \rightarrow \mathcal{P}$ is also given by the same formula (2.25), where $(\cdots)_{0,0}$ now signifies the element in $\operatorname{End}(\mathcal{P})$ corresponding to the transition from $\overbrace{0, \ldots, 0}^{2 n-2}$ to itself in the $\mathcal{M}$ part. From $(3.14)$ one has $T(z) p=$ $z^{w_{1}+\cdots+w_{n-1}+2 w_{n}+w_{-n+1}+\cdots+w_{-1}} T(1) p$ for $p \in \mathcal{P}_{w_{1}, \ldots, w_{-1}}$. The power of $z$ is the total number of particles and antiparticles, for $v_{n}$ represents a bound state of a particle and an antiparticle. As it turns out, the total number is conserved, which implies the commutativity $T(z) T\left(z^{\prime}\right)=T\left(z^{\prime}\right) T(z)$. We concentrate on $T=T(1)$ henceforth.

The propagation operators $\mathcal{K}_{\mu}$ for $\mu= \pm 1, \ldots, \pm(n-1)$ are defined in the same way as (2.28) as the product of $K_{\mu}$ acting locally. This time the local interaction and their amplitudes implied by (3.9) are depicted in Fig. 8.

Here $m_{\mu} \in \mathbb{Z}_{\geq 0}$ is a coordinate in $\left[m_{1}, \ldots, m_{n-1}, m_{-n+1}, \ldots, m_{-1}\right] \in \mathcal{M}$, meaning the number of color $\mu$ particles on the carrier. The top five diagrams are essentially the same as Fig. 2 for $A_{n-1}^{(1)}$ case, where color $\mu$ particles on the carrier (horizontal line) behave according to the presence or absence of another color $\mu$ particle in a local box. (The empty box $-n$ here corresponds to $n$ in $A_{n-1}^{(1)}$ case.) The bottom four vertices are new. The second one there is the pair annihilation of a color $\mu$ particle on the carrier and the antiparticle $-\mu$ in the box to form the bound state $n$. The third one is the pair creation of $\mu$ and $-\mu$ from the bound state 
FiguRE 8. Diagram for $K_{\mu}(\nu \neq \pm \mu, \pm n)$

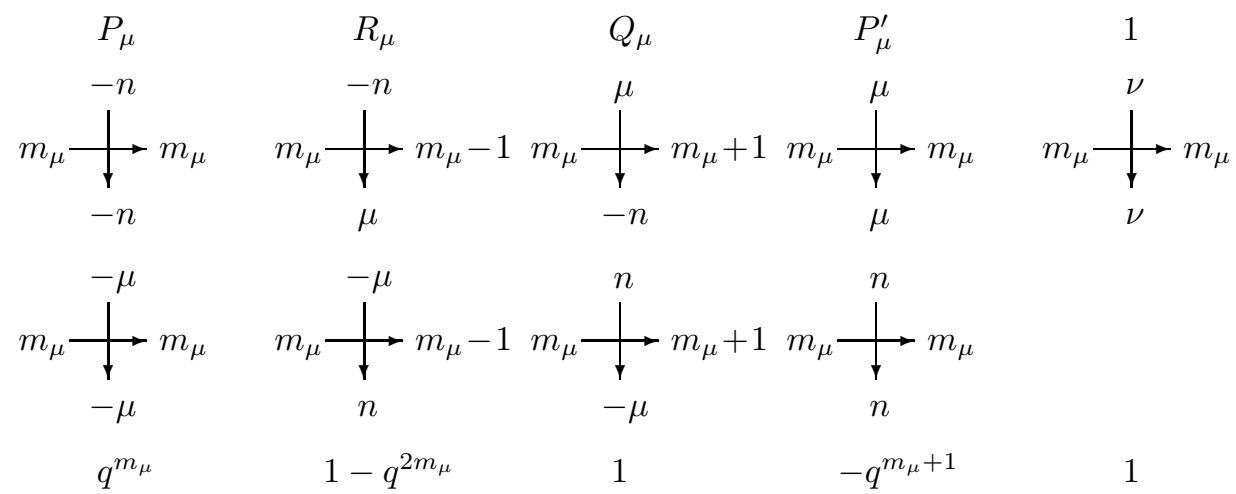

$n$. At $q=0$ the amplitudes for $P_{\mu}$ with $m>0, R_{\mu}$ with $m=0$ and $P_{\mu}^{\prime}$ vanish and the other ones become 1 . As the result they reduce to the deterministic rule that agrees with the one in [HKT3].

As a parallel result with Theorem 2.6, the time evolution of the quantized $D_{n}^{(1)}$ automaton admits the factorization into the propagation operators.

\section{Theorem 3.5.}

$$
T=\mathcal{K}_{-n+1} \cdots \mathcal{K}_{-1} \mathcal{K}_{1} \cdots \mathcal{K}_{n-1}
$$

This is a consequence of Proposition 3.2. It extends a part of the earlier result at $q=0$ based on the crystal basis theory [HKT2, HKT3], where the time evolutions of a class of soliton cellular automata were factorized.

Finally we state properties of the amplitude for $T$. Define the transposition ${ }^{t} T$ of $T$, the subspace $\mathcal{P}_{\text {fin }}$ and the linear function $\mathcal{N}: \mathcal{P}_{\text {fin }} \rightarrow \mathbb{C}$ in the same manner as section 2.7 .

Proposition 3.6. Proposition 2.7 and Proposition 2.9 are both valid also for the quantized $D_{n}^{(1)}$ automaton.

Proof. In view of the factorization of $T$, it is enough to show the claim for any one of the propagation operators, say $\mathcal{K}_{1}$. Namely ${ }^{t} \mathcal{K}_{1}=\mathcal{K}_{1}^{-1}$ and $\mathcal{N}\left(\mathcal{K}_{1}(p)\right)=\mathcal{N}(p)$. Then without a loss of generality one may restrict the space of states to $\mathcal{P}_{w_{1}, \ldots, w_{-1}}$ with all $w_{\mu}$ being zero except $w_{ \pm 1}$ and $w_{n}$. Let $\pi$ be the map that embeds the local states into that for $A_{1}^{(1)}$ as $\pi\left(v_{1}\right)=\pi\left(v_{n}\right)=\bullet$ (a ball) and $\pi\left(v_{-1}\right)=\pi\left(v_{-n}\right)=\circ$ (an empty box), where we have used the notation in section 2.7 for $A_{1}^{(1)}$. Let further $\phi$ be the map sending the pair of local states for $A_{1}^{(1)}$ and $D_{n}^{(1)}$ to that for the latter as

$$
\begin{aligned}
& \phi\left(\circ, v_{1}\right)=v_{-n}, \phi\left(\circ, v_{-1}\right)=v_{-1}, \phi\left(\circ, v_{-n}\right)=v_{-n}, \phi\left(\circ, v_{n}\right)=v_{-1}, \\
& \phi\left(\bullet, v_{1}\right)=v_{1}, \quad \phi\left(\bullet, v_{-1}\right)=v_{n}, \quad \phi\left(\bullet, v_{-n}\right)=v_{1}, \quad \phi\left(\bullet, v_{n}\right)=v_{n} .
\end{aligned}
$$

The componentwise action of these maps will also be denoted by the same symbol. For example, if $p=\cdots \otimes v_{-n} \otimes v_{-1} \otimes v_{-n} \otimes \cdots$ and $p^{\prime}=\cdots \otimes 0 \otimes \bullet \otimes 0 \otimes \cdots$ in the corresponding position, one has $\pi(p)=\cdots \otimes \circ \otimes \circ \otimes \circ \otimes \cdots$ and $\phi\left(p^{\prime}, p\right)=\cdots \phi\left(\circ, v_{-n}\right) \otimes$ $\phi\left(\bullet, v_{-1}\right) \otimes \phi\left(\circ, v_{-n}\right) \otimes \cdots=\cdots \otimes v_{-n} \otimes v_{n} \otimes v_{-n} \otimes \cdots$. Denoting the propagation operator for $A_{1}^{(1)}$ by $\mathcal{K}_{1}^{A}$, one has the embedding $\mathcal{K}_{1}(p)=\phi\left(\mathcal{K}_{1}^{A}(\pi(p)), p\right)$. With 
the aid of this relation, the statements are reduced to the $A_{1}^{(1)}$ case established in section 2.7.

\section{Appendix A. Proof of Proposition 3.1}

The simplifying feature of the limit $x_{-n} \rightarrow \infty$ (3.8) is that one can decompose $w_{\mu \nu}[x \mid y]$ into three parts effectively. To see this suppose $x \in V_{m}$ is in normal order

$$
\left(v_{-1}\right)^{\otimes x_{-1}} \otimes \cdots \otimes\left(v_{-n+1}\right)^{\otimes x_{-n+1}} \otimes\left(v_{-n}\right)^{\otimes x_{-n}} \otimes\left(v_{n-1}\right)^{\otimes x_{n-1}} \otimes \cdots \otimes\left(v_{1}\right)^{\otimes x_{1}} .
$$

Application of (2.2) for $D_{n}^{(1)}$ to this generates a variety of vectors $y=v_{j_{1}} \otimes \cdots \otimes v_{j_{m}}$. However in the limit $x_{-n} \rightarrow \infty$ under consideration, the vectors $v_{1}, \ldots, v_{n}$ are not allowed to appear in the left side of the segment $v_{-n} \otimes \cdots \otimes v_{-n}$ since they acquire the factor of order $q^{x_{-n}}$ in the course of normal ordering. See (3.4). Similarly, $v_{-1}, \ldots, v_{-n+1}$ are forbidden to show up in the right side of $v_{-n} \otimes \cdots \otimes v_{-n}$. In this way $W_{\mu \nu}[x \mid y]$ is effectively decomposed into the right, left and the infinitely large central parts, where the allowed indices are limited to $\{1, \ldots, n-1\},\{-1, \ldots,-n+$ $1\}$ and $-n$, respectively.

Taking the situation into account, we derive $W_{\mu \nu}[x \mid y](3.8)$ in three steps. In Step 1, we compute all the matrix elements $w_{\mu \nu}[x \mid y]$ for $x$ of the form $x=i^{m}=$ $\overbrace{v_{i} \otimes \cdots \otimes v_{i}}^{m}$, which serves as a building block for general $x$. In Step 2, we obtain the limits of $w_{\mu \nu}[x \mid y]$ that are relevant to the three parts separately. In Step 3, we glue the three parts together.

Step 1. 
Lemma A.1. All the matrix elements of the form $w_{j, k}\left[i^{m} \mid y\right]$ are zero except the following:

$w_{i, i}\left[i^{m} \mid i^{m}\right]=\left(1-q^{m+1} z\right)\left(1-q^{m-1} \xi z\right) \quad \forall i$,

$w_{-i,-i}\left[i^{m} \mid i^{m}\right]=\left(q^{m-1}-z\right)\left(q^{m+1}-\xi z\right) \quad \forall i$,

(A.3)

$w_{j, j}\left[i^{m} \mid i^{m}\right]=q\left(q^{m-1}-z\right)\left(1-q^{m-1} \xi z\right) \quad j \neq \pm i \forall i$,

$w_{j, i}\left[i^{m} \mid i^{m-1}, j\right]=\left(1-q^{2 m}\right)\left(1-q^{m-1} \xi z\right) \times\left\{\begin{array}{ll}1 & i \succ j, j \neq \pm i \\ z & i \prec j, j \neq \pm i\end{array} \quad \forall i\right.$

$w_{-i, j}\left[i^{m} \mid-j, i^{m-1}\right]=(-1)^{i+j+1}\left(1-q^{2 m}\right)\left(q^{m-1}-z\right) q^{\bar{j}+\bar{i}-2} \times\left\{\begin{array}{ll}z & 1 \preceq j \prec-i \\ \xi^{-1} & -i \prec j \preceq-1\end{array} \quad \forall i\right.$,

$w_{-i, i}\left[i^{m} \mid i^{m-2}, j,-j\right]=(-1)^{i+j+1} q^{n-j-1}\left(1-q^{2 m}\right)\left(1-q^{2 m-2} \xi\right) z \quad i= \pm n, 1 \leq j \leq n-1$,

$$
\begin{aligned}
& w_{-i, i}\left[i^{m} \mid-i, i^{m-1}\right] \\
& =\left\{\begin{array}{ll}
q^{m-1}\left(1-q^{2 m}\right)\left(1-q^{m-1} \xi z+q^{2 i-1-m}\left(z-q^{m-1}\right)\right) z & 1 \leq i \leq n-1 \\
\left(1-q^{2 m}\right)\left(1-q^{m-1} \xi z+q^{2 i+1+m} \xi\left(z-q^{m-1}\right)\right) & -n+1 \preceq i \preceq-1
\end{array} \quad i \neq \pm n,\right.
\end{aligned}
$$

$$
\begin{aligned}
& w_{-i, i}\left[i^{m} \mid i^{m-2}, j,-j\right] \\
& = \begin{cases}(-1)^{i+j+1} q^{i-j-1}\left(1-q^{2 m}\right)\left(1-q^{2 m-2}\right)\left(1-q^{m-1} \xi z\right) z & 1 \leq i \leq n-1,1 \leq j<i \\
(-1)^{i+j} q^{i-j+1} \xi\left(1-q^{2 m}\right)\left(1-q^{2 m-2}\right)\left(q^{m-1}-z\right) & -n+1 \preceq i \preceq-1,1 \leq j<|i| .\end{cases}
\end{aligned}
$$

In these formulas for $w_{j, k}\left[i^{m} \mid y\right], y$ should be understood as a normal ordered vector in $V_{m}$ having the specified contents of the letters.

Sketch of the proof. The first four, (A.1)-(A.4), are straightforward to check. The other formulas (A.5)-(A.8) are shown in this order by induction on $m$. Here we illustrate it for (A.8). Let us write the $R$-matrix (3.2) as $R(z)=\sum_{i, j, k, l} r[i, k ; j, l](z) E_{j i} \otimes$ $E_{l k}$. For simplicity $w_{j, k}\left[i^{m} \mid y\right](z)$ will be denoted by $w_{j, k}[y](z)$. We treat the case $1 \leq j<i \leq n-1$. The result (A.8) for $m=2$ can be checked directly. Assume (A.1)-(A.8) up to $m$. The fusion construction leads to the following recursion 
relation for $m \geq 3$ :

$$
\begin{aligned}
& w_{-i, i}\left[i^{m-1}, j,-j\right](z) a\left(z q^{m-2}\right) \\
& =\underline{q} r[i, i ; i, i]\left(z q^{m}\right) w_{-i, i}\left[i^{m-2}, j,-j\right]\left(z q^{-1}\right) \\
& +\sum_{\alpha \neq i, \alpha=j+1}^{n-1} \underline{(-1)^{1+\alpha+j}\left(1-q^{2}\right) q^{\alpha-j+m-1}} r[i, \alpha ; \alpha, i]\left(z q^{m}\right) w_{-i, \alpha}\left[i^{m-1},-\alpha\right]\left(z q^{-1}\right) \\
& \left.+\underline{(-1)^{i+j+1}\left(1-q^{2}\right) q^{i-j+m-1} r} r i, i ; i, i\right]\left(z q^{m}\right) w_{-i, i}\left[i^{m-1},-i\right]\left(z q^{-1}\right) \\
& +\underline{q^{m+1}} r[i, j ; j, i]\left(z q^{m}\right) w_{-i, j}\left[i^{m-1},-j\right]\left(z q^{-1}\right) \\
& +r[i,-j ;-j, i]\left(z q^{m}\right) w_{-i,-j}\left[i^{m-1}, j\right]\left(z q^{-1}\right) \\
& +\underline{(-1)^{n+j+1} q^{n-j+m-1}}\left(r[i,-n ;-n, i]\left(z q^{m}\right) w_{-i,-n}\left[i^{m-1}, n\right]\left(z q^{-1}\right)\right. \\
& \left.+r[i, n ; n, i]\left(z q^{m}\right) w_{-i, n}\left[i^{m-1},-n\right]\left(z q^{-1}\right)\right),
\end{aligned}
$$

where the underlined factors come from the normal ordering. To check that (A.1)(A.8) satisfy this is easy.

Step 2. As explained in the beginning of the appendix, we investigate the three parts that constitute the limit $W_{\mu \nu}[x \mid y]$ separately. First we consider the right part.

Lemma A.2. Set $w_{\mu \nu}^{\prime}[x \mid y]=w_{\mu \nu}^{\prime}[x \mid y](z)=w_{\mu \nu}[x \mid y] / a(z)$ and $m_{1}=x_{1, n-1}$. Suppose $x$ and $y$ have the form $x=\left[x_{1}, \ldots, x_{n-1}, 0, \ldots, 0\right]$ and $y=\left[y_{1}, \ldots, y_{n-1}, y_{-n}, 0, \ldots, 0\right]$, respectively. Then the nonzero case of the limit $\lim _{z \rightarrow \infty} w_{\mu \nu}^{\prime}[x \mid y]$ is given by

$$
\begin{aligned}
& w_{ \pm j, \pm j}^{\prime}[x \mid x] \rightarrow q^{-m_{1} \pm x_{j}}(1 \leq j \leq n), \\
& w_{j, k}^{\prime}[x \mid x+(j)-(k)] \rightarrow-\left(1-q^{2 x_{k}}\right) q^{-m_{1}-1+x_{k+1, j-1}} \quad(1 \leq k<j \leq n-1), \\
& w_{-j,-k}^{\prime}[x \mid x-(j)+(k)] \rightarrow(-1)^{j+k}\left(1-q^{2 x_{j}}\right) q^{-m_{1}+j-k-x_{j, k}} \quad(1 \leq j<k \leq n-1), \\
& w_{-n, k}^{\prime}[x \mid x+(-n)-(k)] \rightarrow-\left(1-q^{2 x_{k}}\right) q^{-1-x_{1, k}}(1 \leq k \leq n-1), \\
& w_{-j, n}^{\prime}[x \mid x-(j)+(-n)] \rightarrow(-1)^{j+n}\left(1-q^{2 x_{j}}\right) q^{-m_{1}+j-n-x_{j, n-1}}(1 \leq j \leq n-1) .
\end{aligned}
$$

Sketch of the proof. We illustrate the derivation of the second case. From the fusion construction one gets

$$
\begin{aligned}
& w_{j, k}^{\prime}[x \mid x+(j)-(k)]\left(z q^{m_{1}-1}\right)=q^{x_{k+1, j-1}} \frac{w_{j, k}\left[k^{x_{k}} \mid k^{x_{k}-1}, j\right]\left(z q^{x_{k}-1}\right)}{a\left(z q^{2\left(x_{k}-1\right)}\right)} \\
& \times\left(\prod_{i=1}^{k-1} \frac{w_{k, k}\left[i^{x_{i}} \mid i^{x_{i}}\right]\left(z q^{2 x_{k}+2 x_{1, i-1}+x_{i}-1}\right)}{a\left(z q^{2 x_{k}+2\left(x_{1, i}-1\right)}\right)} \prod_{i=k+1}^{n-1} \frac{w_{k, k}\left[i^{x_{i}} \mid i^{x_{i}}\right]\left(z q^{2 x_{1, i-1}+x_{i}-1}\right)}{a\left(z q^{2\left(x_{1, i}-1\right)}\right)}\right),
\end{aligned}
$$

where the factor $q^{x_{k+1, j-1}}$ is due to normal ordering. Substituting (A.3) and (A.4), one finds that this tends to the desired form in the limit $z \rightarrow \infty$.

Next we deal with the central part. 
Lemma A.3. Nonzero limit $q^{x_{-n}} \rightarrow 0$ of $w_{\mu \nu}\left[(-n)^{x_{-n}} \mid y\right]$ is given by

$$
\begin{aligned}
& w_{n, n}\left[(-n)^{x_{-n}} \mid(-n)^{x_{-n}}\right] \rightarrow \xi z^{2}, \\
& w_{-n,-n}\left[(-n)^{x_{-n}} \mid(-n)^{x_{-n}}\right] \rightarrow 1, \\
& w_{n,-n}\left[(-n)^{x_{-n}} \mid-j,(-n)^{x_{-n}-2}, j\right] \rightarrow(-1)^{j+n+1} q^{n-j-1} z, \\
& w_{n, j}\left[(-n)^{x_{-n}} \mid-j,(-n)^{x_{-n}-1}\right] \rightarrow(-1)^{j+n} q^{n+j-2} z^{2}, \\
& w_{n,-j}\left[(-n)^{x_{-n}} \mid(-n)^{x_{-n}-1}, j\right] \rightarrow(-1)^{j+n} q^{n-j} z, \\
& w_{ \pm j, \pm j}\left[(-n)^{x_{-n}} \mid(-n)^{x_{-n}}\right] \rightarrow-q z, \\
& w_{j,-n}\left[(-n)^{x_{-n}} \mid(-n)^{x_{-n}-1}, j\right] \rightarrow 1, \\
& w_{-j,-n}\left[(-n)^{x_{-n}} \mid-j,(-n)^{x_{-n}-1}\right] \rightarrow z,
\end{aligned}
$$

where $1 \leq j \leq n-1$.

Proof. Straightforward calculation based on Lemma A.1.

Finally for the left part, the following is verified similarly to Lemma A.2.

Lemma A.4. Suppose $x$ and $y$ have the form $x=\left[0, \ldots, 0, x_{-n+1}, \ldots, x_{-1}\right]$ and $y=\left[0, \ldots, 0, y_{-n}, y_{-n+1}, \ldots, y_{-1}\right]$. Then the nonzero case of the limit $\lim _{z \rightarrow 0} w_{\mu \nu}[x \mid y]$ is given by

$w_{ \pm j, \pm j}[x \mid x] \rightarrow q^{m_{2} \pm x_{-j}}(1 \leq j \leq n)$,

$w_{j, k}[x \mid x-(-j)+(-k)] \rightarrow(-1)^{j+k+1}\left(1-q^{2 x_{-j}}\right) q^{m_{2}+k-j-1+x_{-j-1,-k+1}}(1 \leq j<k \leq n)$,

$w_{-j,-k}[x \mid x+(-j)-(-k)] \rightarrow\left(1-q^{2 x_{-k}}\right) q^{m_{2}-x_{-k,-j}}(1 \leq k<j \leq n)$,

where $m_{2}=x_{-1,-n+1}$.

Step 3. We demonstrate the gluing procedure with two examples. First we derive the 4th case in Proposition 3.1, $W_{i, l}[x \mid x+(i)-(j)-(-j)+(-l)]$. This is calculated as the simple product of the three parts:

$$
\begin{aligned}
& w_{i, j}^{\prime}[x \mid x+(i)-(j)]\left(z q^{-m+m_{1}}\right) w_{j, j}\left[(-n)^{x_{-n}} \mid(-n)^{x_{-n}}\right]\left(z q^{m_{1}-m_{2}}\right) \\
& \quad \times w_{j, l}\left[x^{\prime} \mid x^{\prime}-(-j)+(-l)\right]\left(z q^{m-m_{2}}\right),
\end{aligned}
$$

which is nonzero for $1 \leq j \leq \min (i, l)$. For $j<i<l$, it is calculated by multiplying the second one in Lemma A.2, the 6th of Lemma A.3 and the second of Lemma A.4, leading to

$$
\begin{aligned}
& -\left(1-q^{2 x_{j}}\right) q^{-m_{1}-1+x_{j+1, i-1}} \times\left(-z q^{1+m_{1}-m_{2}}\right) \times(-1)^{j+l+1}\left(1-q^{2 x_{-j}}\right) q^{m_{2}+l-j-1+x_{-j-1,-l+1}} \\
& =(-1)^{j+l+1} z\left(1-q^{2 x_{j}}\right)\left(1-q^{2 x_{-j}}\right) q^{l-j-1+x_{j+1, i-1}+x_{-j-1,-l+1}} .
\end{aligned}
$$

This agrees with the sought result. Second we consider the 9th case in Proposition $3.1, W_{i,-k}[x \mid x+(i)-(-k)]$. This matrix element is obtained by collecting several contributions as

$$
\begin{aligned}
& \left(\frac{q^{x_{i+1, n-1}}}{w_{i, i}^{\prime}}[x \mid x]\left(z q^{-m+m_{1}}\right) w_{i,-n}\left[(-n)^{x_{-n}} \mid(-n)^{x_{-n}-1}, i\right]\left(z q^{m_{1}-m_{2}}\right)\right. \\
& \left.+\sum_{j=1}^{i-1} \underline{q^{x_{j+1, n-1}+1}} w_{i, j}^{\prime}[x \mid x+(i)-(j)]\left(z q^{-m+m_{1}}\right) w_{j,-n}\left[(-n)^{x_{-n}} \mid(-n)^{x_{-n}-1}, j\right]\left(z q^{m_{1}-m_{2}}\right)\right) \\
& \times w_{-n,-k}\left[x^{\prime} \mid x^{\prime}-(-k)+(-n)\right]\left(z q^{m-m_{2}}\right),
\end{aligned}
$$


where we have set $x=\left[x_{1}, \ldots, x_{n-1}, 0, \ldots, 0\right]$ and $x^{\prime}=\left[0, \ldots, 0, x_{-n+1}, \ldots, x_{-1}\right]$. The underlined factors come from normal ordering. In the limit $x_{-n} \rightarrow \infty$, this is evaluated by using the first two of Lemma A.2, the 7th of Lemma A.3 and the last of Lemma A.4 as

$$
\left(q^{x_{i, n-1}}-\sum_{j=1}^{i-1}\left(1-q^{2 x_{j}}\right) q^{x_{j+1, i-1}+x_{j+1, n-1}}\right)\left(1-q^{2 x_{-k}}\right) q^{m_{2}-x_{-k,-n+1}-m_{1}}
$$

The sum leads to the result $\left(1-q^{2 x_{-k}}\right) q^{x_{1, i-1}+x_{-1,-k+1}}$.

\section{Appendix B. Proof of Proposition 3.2}

Let $L_{n}\left[\begin{array}{ll}P^{\prime} & R \\ Q & P\end{array}\right]$ be the $L$ operator $L(z)$ for $A_{n-1}^{(1)}$ with $z=1$ defined in (2.12). The $L$ operator with $P_{i}$ and $P_{i}^{\prime}$ interchanged for all $i \in\{1, \ldots, n-1\}$ will be denoted by $L_{n}\left[\begin{array}{ll}P & R \\ Q & P^{\prime}\end{array}\right]$. A similar convention is applied also for the other interchanges like $R_{i} \leftrightarrow Q_{i}$, etc. A matrix $\bar{L}_{n}[\cdots]$ is the one obtained from $L_{n}[\cdots]$ by changing $X_{i}\left(X=P, P^{\prime}, Q, R\right)$ into $X_{-i}$ for all $i \in\{1, \ldots, n-1\}$. Matrices $L_{n}^{+}[\cdots]$ and $\bar{L}_{n}^{+}[\cdots]$ are the ones obtained from $L_{n}[\cdots]$ and $\bar{L}_{n}[\cdots]$ respectively by the replacement $X_{ \pm i} \rightarrow X_{ \pm(i+1)}$ for all $i \in\{1, \ldots, n-1\}$. For any square matrix $M$ we let $\tilde{M}$ denote the one obtained by reversing the order of rows and columns simultaneously.

\section{Lemma B.1.}

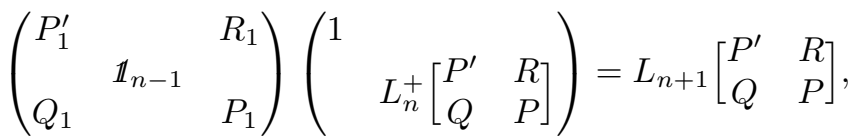

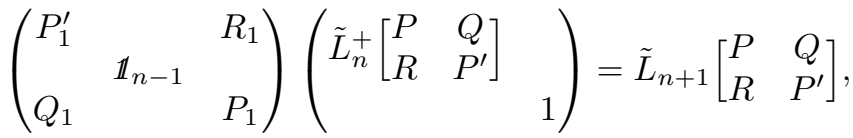

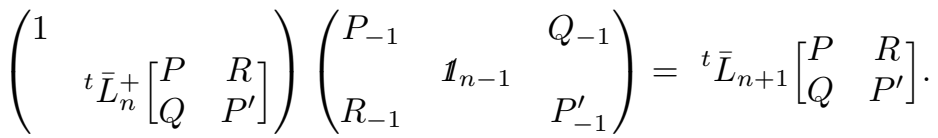

$$
\begin{aligned}
& \left(\begin{array}{ccc}
{ }^{t} \tilde{\bar{L}}_{n}^{+}\left[\begin{array}{cc}
P^{\prime} & Q \\
R & P
\end{array}\right] & \\
& & 1
\end{array}\right)\left(\begin{array}{ccc}
P_{-1} & & Q_{-1} \\
& \mathbb{1}_{n-1} & \\
R_{-1} & & P_{-1}^{\prime}
\end{array}\right)={ }^{t} \tilde{\bar{L}}_{n+1}\left[\begin{array}{cc}
P^{\prime} & Q \\
R & P
\end{array}\right] .
\end{aligned}
$$

Here ${ }^{t}$ means the transposition.

Proof. The first relation is just (2.16). The second relation is obtained from the first one by taking ${ }^{\sim}$ and the interchanges $P \leftrightarrow P^{\prime}, Q \leftrightarrow R$. See Remark 2.3. The third relation follows from the first one by ${ }^{t-}$ and $P \leftrightarrow P^{\prime}$. The last one follows from the third one by $\sim$ and $P \leftrightarrow P^{\prime}, Q \leftrightarrow R$. 


\section{Lemma B.2.}

$$
\begin{aligned}
& K_{1} \cdots K_{n-1}=\rho\left(\begin{array}{ccc}
L_{n}\left[\begin{array}{cc}
P^{\prime} & R \\
Q & P
\end{array}\right] & & \\
& & \tilde{L}_{n}\left[\begin{array}{cc}
P & Q \\
R & P^{\prime}
\end{array}\right]
\end{array}\right) \rho,
\end{aligned}
$$

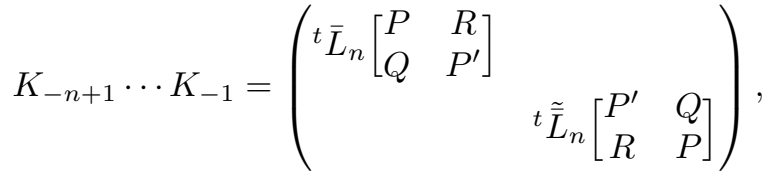

where $\rho \in \operatorname{End}(V)$ denotes the interchange $v_{n} \leftrightarrow v_{-n}$.

Proof. We use induction on $n$. The $n=3$ case is checked by a direct calculation. Assume (B.1) and (B.2) are fulfilled up to $n$. Then the left hand side of (B.1) for $n+1$ is

$$
\begin{aligned}
& K_{1} K_{2} \cdots K_{n}
\end{aligned}
$$

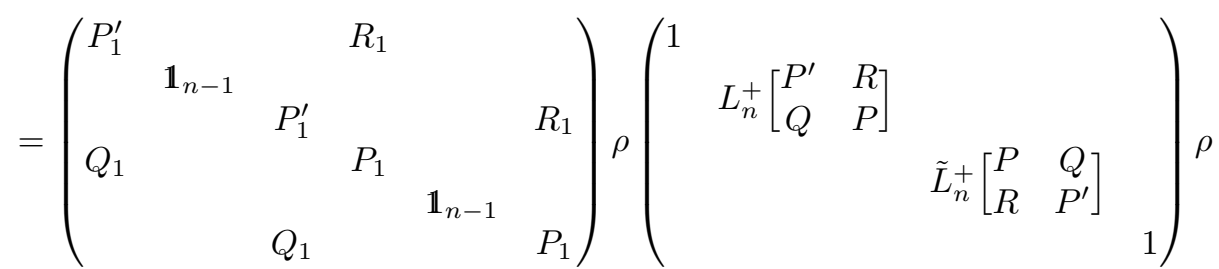

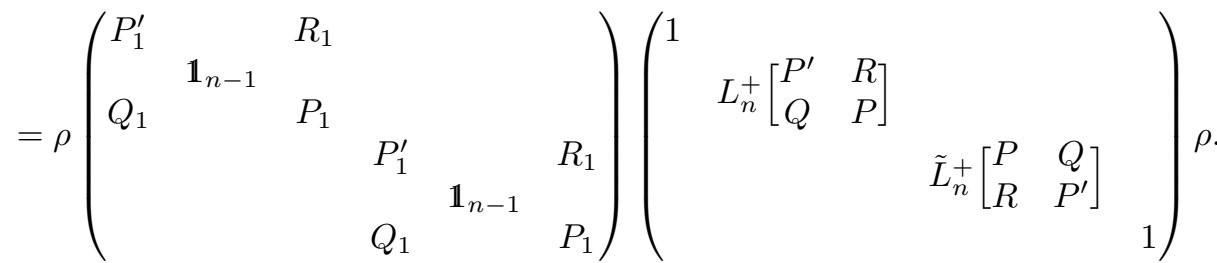

Owing to the first two relations in Lemma B.1, this coincides with the right hand side of (B.1) for $n+1$. Similarly the induction assumption leads to the following expression for the left hand side of (B.2) for $n+1$ :

$$
\begin{aligned}
& K_{-n} K_{-n+1} \cdots K_{-1}
\end{aligned}
$$

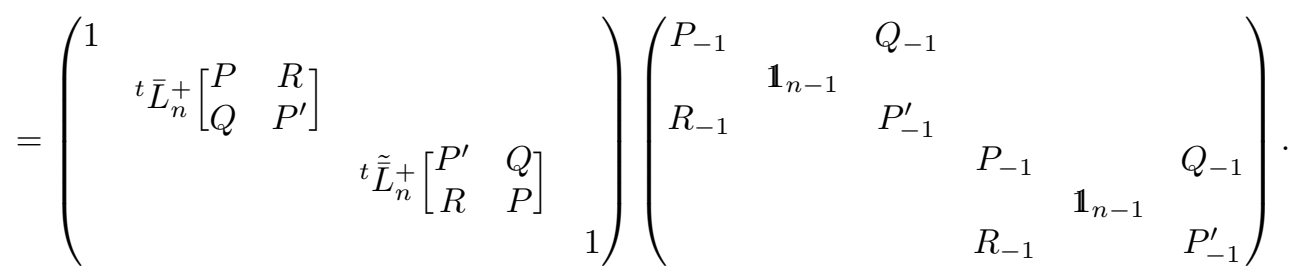

Again the product can be computed by using the latter two relations in Lemma B.1, yielding the right hand side of (B.2) for $n+1$. This completes the induction.

Proof of Proposition 3.2 The product $K_{-n+1} \cdots K_{-1} D(z) K_{1} \cdots K_{n-1}$ can be calculated by using Lemma B.2, (3.14) and (2.12). The result agrees with the $L(z)$ defined in section 3.3. 


\section{ACKNOWLEDGEMENTS}

The authors thank Taichiro Takagi and Yasuhiko Yamada for discussion. A.K. thanks Murray Batchelor, Vladimir Bazhanov, Vladimir Mangazeev and Sergey Sergeev for a warm hospitality at the Australian National University during his stay in March 2004. A.K. and M.O. are partially supported by Grand-in-Aid for Scientific Research JSPS No.15540363 and No.14540026, respectively from Ministry of Education, Culture, Sports, Science and Technology of Japan.

\section{REFERENCES}

[Bax] R. J. Baxter, Exactly solved models in statistical mechanics, Academic Press, London (1982).

[B] V. V. Bazhanov, Integrable quantum systems and classical Lie algebras, Comm. Math. Phys. 113 (1987) 471-503.

[BS] V. V. Bazhanov and Yu. G. Stroganov, Chiral Potts model as a descendant of the six-vertex model, J. Stat. Phys. 59 (1990) 799-817.

[FOY] K. Fukuda, M. Okado, Y. Yamada, Energy functions in box ball systems, Int. J. Mod. Phys. A 15 (2000) 1379-1392.

[HHIKTT] G. Hatayama, K. Hikami, R. Inoue, A. Kuniba, T. Takagi and T. Tokihiro, The $A_{M}^{(1)}$ Automata related to crystals of symmetric tensors, J. Math. Phys. 42 (2001) 274-308.

[HKT1] G. Hatayama, A. Kuniba, and T. Takagi, Soliton cellular automata associated with crystal bases, Nucl. Phys. B577[PM] (2000) 619-645.

[HKT2] G. Hatayama, A. Kuniba, and T. Takagi, Factorization of combinatorial $R$ matrices and associated cellular automata, J. Stat. Phys. 102 (2001) 843-863.

[HKT3] G. Hatayama, A. Kuniba, and T. Takagi, Simple algorithm for factorized dynamics of $\mathfrak{g}_{n}$-automaton, J. Phys. A: Math. Gen.34 (2001) 10697-10705.

[HKOTY] G. Hatayama, A. Kuniba, M. Okado, T. Takagi and Y. Yamada, Scattering rules in soliton cellular automata associated with crystal bases, Contemporary Math. 297 (2002) 151-182.

[HIK] K. Hikami, R. Inoue and Y. Komori, Crystallization of the Bogoyavlensky lattice, J. Phys. Soc. Jpn. 68 (1999) 2234-2240.

[J] M. Jimbo, Quantum $R$ matrix for the generalized Toda system, Comm. Math. Phys. 102 (1986) 537-547.

[KKM] S-J. Kang, M. Kashiwara and K. C. Misra, Crystal bases of Verma modules for quantum affine Lie algebras, Compositio Math. 92 (1994) 299-325.

[KT] S. M. Khoroshkin and V. N. Tolstoy, Universal $R$-matrix for quantized (super) algebras, Commun. Math. Phys. 141 (1991) 599-617.

[KR] A. N. Kirillov and N. Yu. Reshetikhin, $q$-Weyl group and a multiplicative formula for universal R-matrices, Commun. Math. Phys. 134 (1990) 421-431.

[KRS] P. P. Kulish, N. Yu. Reshetikhin and E. K. Sklyanin, Yang-Baxter equations and representation theory. I, Lett. Math. Phys. 5 (1981) 393-403.

[KTT] A. Kuniba, T. Takagi and A. Takenouchi, Factorization, reduction and embedding in integrable cellular automata, J. Phys. A 37 (2004) 1691-1709.

[KOTY1] A. Kuniba, M. Okado, T. Takagi and Y. Yamada, Geometric crystal and tropical $R$ for $D_{n}^{(1)}$, Int. Math. Res. Notices 48 (2003) 2565-2620.

[KOTY2] A. Kuniba, M. Okado, T. Takagi and Y. Yamada, Tropical $R$ and tau functions, Commun. Math. Phys. 245 (2004) 491-517.

[NY] A. Nakayashiki and Y. Yamada, Kostka polynomials and energy functions in solvable lattice models, Selecta Mathematica, New Ser. 3 (1997) 547-599.

[S] Ya. S. Soibelman, Quantum Weyl group and some of its applications, Rend. Circ. Mat. Palermo Suppl. 26 (1991) 233-235.

[T] D. Takahashi, On some soliton systems defined by using boxes and balls, Proceedings of the International Symposium on Nonlinear Theory and Its Applications (NOLTA '93), (1993) $555-558$.

[TM] D. Takahashi and J. Matsukidaira, Box and ball system with a carrier and ultra-discrete modified KdV equation, J. Phys. A 30 (1997) L733 - L739. 
[TS] D. Takahashi and J. Satsuma, A soliton cellular automaton, J. Phys. Soc. Jpn. 59 (1990) $3514-3519$.

[TTMS] T. Tokihiro, D. Takahashi, J. Matsukidaira and J. Satsuma, From soliton equations to integrable cellular automata through a limiting procedure, Phys. Rev. Lett. 76, (1996) $3247-3250$.

Research Institute for Mathematical Sciences, Kyoto University, Kyoto 606-8502, JAPAN

E-mail address: reiiy@kurims.kyoto-u.ac.jp

Institute of Physics, University of TOKyo, TOKyo 153-8902, JAPAN

E-mail address: atsuo@gokutan.c.u-tokyo.ac.jp

Division of Mathematical Science, Graduate School of Engineering Science, Osaka UNIVERSITY, OSAKA 560-8531, JAPAN

E-mail address: okado@sigmath.es.osaka-u.ac.jp 